\title{
Upper Semicontinuity of Random Attractor for a Kirchhoff Type Suspension Bridge Equation with Strong Damping and White Noise
}

\author{
Ling $\mathrm{Xu}^{*}$ and Qiaozhen Ma
}

\begin{abstract}
This paper is devoted to the well-posedness and long-time behavior of a stochastic Kirchhoff type suspension bridge equation with strong damping. The existence of the random attractor for a Kirchhoff type suspension bridge equation with white noise is established. Moreover, the upper semicontinuity of random attractors is also provided when the coefficient of random term approaches zero.
\end{abstract}

\section{Introduction}

Lazer and McKenna presented the following suspension bridge equations in $[9$ as a new problem of nonlinear analysis

$$
u_{t t}+\triangle^{2} u+\alpha u_{t}+k u^{+}=w(x, t)+f(x)
$$

and studied the large-amplitude periodic oscillations of solutions. The existence of the periodic solutions and numerical simulations were investigated in [1,8,14. We first achieved the existence of global attractors for the coupled suspension bridge equations [11]. From then on, the universal compact attractors for both the single and coupled suspension bridge equations have been studied systematically by many authors, for instance $10,12,15,17,24$ and reference therein. Combining the earlier ideas of Woinowsky-Krieger on the extensible elastic beam 21] with (1.1), that is, taking into account the midplane stretching of road bed due to its elongation, Bochicchio etc. [3] arrived at the following equation

$$
u_{t t}+\triangle^{2} u+\alpha u_{t}+\left(p-\|\nabla u\|_{L^{2}(U)}^{2}\right) \triangle u+k u^{+}=f(x),
$$

Received February 5, 2019; Accepted July 28, 2019.

Communicated by Cheng-Hsiung Hsu.

2010 Mathematics Subject Classification. 60H15, 35Q35, 35B40.

Key words and phrases. a Kirchhoff type suspension bridge equation, random dynamical system, random attractor, white noise, upper semicontinuity.

This work is supported by the NSF of China (11361053), the NSF of Gansu Province (17JR5RA069), the University Project of Gansu Province (2017B-90) and the Project of Northwest Normal University (NWNU-LKQN-16-16, NWNU-LKQN-18-14).

*Corresponding author. 
moreover, they proved the existence of global attractors of optimal regularity as $p \in \mathbb{R}$. If we consider the effect of strong damping and white noise in (1.2), then the following equation is derived

$$
u_{t t}+\triangle^{2} u+\triangle^{2} u_{t}+\left(p-\|\nabla u\|_{L^{2}(U)}^{2}\right) \triangle u+k u^{+}+f(u, x)=g(x, t)+a q(x) \dot{W}(t)
$$

with the both ends of simple supported boundary

$$
u(t, \tau, x)=\triangle u(t, \tau, x)=0, \quad x \in \partial U, t \geq \tau,
$$

and the initial value condition

$$
u(t, \tau, x)=u_{\tau}(t, \tau, x), \quad u_{t}(t, \tau, x)=u_{1, \tau}(t, \tau, x), \quad t \leq \tau,
$$

where $U$ is an open bounded subset of $\mathbb{R}^{2}$ with smooth boundary $\partial U$, the unknown $u=u(t, \tau, x)$ denotes the downward deflection of the bridge in the vertical plane, and $u^{+}$ stands for its positive part, namely,

$$
u^{+}= \begin{cases}u & \text { if } u \geq 0 \\ 0 & \text { if } u<0\end{cases}
$$

$k>0$ is the spring constant, $-k u^{+}$models a restoring force due to the cables, which is different from zero only when they are being stretched. The real constant $p$ accounts for the axial force acting at the end of the road bed of the bridge in the reference configuration. Namely, $p$ is positive when the bridge is compressed, negative when stretched, $a \in \mathbb{R} . q(x)$ is not identically equal to zero, $f$ is a nonlinear function satisfying certain conditions. $g(\cdot, t) \in C_{b}\left(\mathbb{R}, H_{0}^{1}(U)\right), C_{b}\left(\mathbb{R}, H_{0}^{1}(U)\right)$ denotes the set of continuous bounded functions from $\mathbb{R}$ into $H_{0}^{1}(U) . \dot{W}$ is the derivative of a one-dimensional two-sided real-valued Wiener process $W(t)$, the white noise described by $q(x) \dot{W}$. However, in the real world, small irregularity has to be considered. Thus it is necessary to take account of the equation with a random force. For this purpose, we will focus on the existence and the upper semicontinuity of random attractors for $1.3-1.5$.

The classical results of global attractors for the deterministic dynamical system is clearly not true for RDS. For a dynamical system perturbed by a noise, the difficulty is of course in the fact that how to establish a compact random invariant set. The authors introduce the concept of random attractors for the infinite-dimensional random dynamical systems in [2,5], and these abstract results have been successfully applied into other stochastic dissipative partial differential equations $6,7,13,22,23$ and references therein. Therefore, we will use the methods established by Crauel, Flandoli, Arnold and Wang etc. in $[2,5,20]$ to establish the existence of random attractors for $1.3-(1.5)$, and then we establish the upper semicontinuity of random attractors when the coefficient $a$ in $(1.3)$ 
approaches zero. Moreover, another important ingredient in the argument is the parameter $p \in \mathbb{R}$, which gives difficulties for estimation.

We assume that the nonlinearity $f(\cdot, x) \in C^{2}(\mathbb{R}, \mathbb{R})$ is subject to the following growth and dissipation conditions: for $x \in U$ and $u \in \mathbb{R}$,

(a) Growth conditions:

$$
|f(u, x)| \leq C_{0}|u|^{\gamma}+\phi_{1}(x), \quad \gamma \geq 1, \phi_{1}(x) \in L^{2}(U)
$$

where $C_{0}$ is a positive constant.

(b) Dissipation conditions:

$$
\begin{gathered}
F(u, x):=\int_{0}^{u} f(s, x) d s \geq C_{1}|u|^{\gamma+1}-\phi_{2}(x), \quad \gamma \geq 1, \phi_{2}(x) \in L^{1}(U), \\
u f(u, x) \geq C_{2} F(u, x)-\phi_{3}(x), \quad \phi_{3}(x) \in L^{1}(U),
\end{gathered}
$$

where $C_{1}, C_{2}$ are positive constants.

This paper is arranged as follows: In Section 2, we introduce some definition and useful lemmas on random dynamical systems. In Section 3 , we establish the existence and uniqueness of the solution corresponding to the system (3.3), which can generates a random dynamical systems. In Section 4, we obtain a compact measurable pullback attracting set and the existence of random attractor for equation (3.3). Finally, the upper semicontinuity of random attractors is proved in Section 5 as $a \rightarrow 0$.

In this paper, the letters $C_{i}(i \in \mathbb{N})$ below are generic positive constants which do not depend on $\omega, \tau$ and $t$.

\section{Preliminaries}

In this section, we introduce some definitions and useful lemmas, which is the key technique to establish the existence and upper semicontinuity of the random attractor, which are from $2,4,5,19,20$.

Let $\left(X,\|\cdot\|_{X}\right)$ be a separable Hilbert space with Borel $\sigma$-algebra $\mathcal{B}(X)$, and let $(\Omega, \mathcal{F}, \mathbb{P})$ be a probability space, where

$$
\Omega=\{\omega(t) \in C(\mathbb{R}, \mathbb{R}): \omega(0)=0\},
$$

$\mathcal{F}$ is the Borel $\sigma$-algebra induced by the compact-open topology of $\Omega$, and $\mathbb{P}$ is a Wiener measure. In addition, we write $W(t, \omega)=\omega(t)$ and define

$$
\theta_{t} \omega(\cdot)=\omega(\cdot+t)-\omega(t), \quad t \in \mathbb{R}, \omega \in \Omega .
$$


Then $\theta_{t}: \Omega \rightarrow \Omega, t \in \mathbb{R}$ is a family of measure preserving and ergodic transformations such that $(t, \omega) \mapsto \theta_{t} \omega$ is measurable, $\theta_{0}=$ id and $\theta_{t+s}=\theta_{t} \theta_{s}$ for all $t, s \in \mathbb{R}$. The flow $\theta_{t}$ together with the probability space $\left(\Omega, \mathcal{F}, \mathbb{P},\left(\theta_{t}\right)_{t \in \mathbb{R}}\right)$ is called as an ergodic metric dynamical system.

Definition 2.1. Let $\left(\Omega, \mathcal{F}, \mathbb{P},\left(\theta_{t}\right)_{t \in \mathbb{R}}\right)$ be a measurable dynamical system. Suppose that the mapping $\Phi: \mathbb{R}^{+} \times \Omega \times X \rightarrow X$ is $\left(\mathcal{B}\left(\mathbb{R}^{+}\right) \times \mathcal{F} \times \mathcal{B}(X), \mathcal{B}(X)\right)$-measurable and satisfies the following properties:

(i) $\Phi(0, \tau, \omega, \cdot) x=x, \tau \in \mathbb{R}, x \in X$ and $\omega \in \Omega$;

(ii) $\Phi(t+s, \tau, \omega, \cdot)=\Phi\left(t, \tau+s, \theta_{s} \omega\right) \circ \Phi(s, \tau, \omega, \cdot)$ for all $t, s \in \mathbb{R}^{+}, \tau \in \mathbb{R}, x \in X$ and $\omega \in \Omega$.

Then $\Phi$ is called a random dynamical system (RDS). Moreover, $\Phi$ is called a continuous $\operatorname{RDS}$ if $\Phi$ is continuous with respect to $x$ for $t \geq 0, \tau \in \mathbb{R}$ and $\omega \in \Omega$.

Let $D$ be a family if some subsets of $X$ which is parameterized by $(\tau, \omega) \in \mathbb{R} \times \Omega$ : $D=\{D(\tau, \omega) \subseteq X: \tau \in \mathbb{R}, \omega \in \Omega\}$. The family $D$ is measurable with respect to $\mathcal{F}$ in $\Omega$ if the set $D(\tau, \omega)$ is a closed nonempty subset of $X$ for all $\tau \in \mathbb{R}$ and $\omega \in \Omega$, and the mapping $\omega \in \Omega \rightarrow d(x, D(\tau, \omega))$ is $\left(\mathcal{F}, \mathcal{B}\left(\mathbb{R}^{+}\right)\right.$-measurable for every fixed $x \in X$.

Definition 2.2. A family $B=\{B(\tau, \omega) \subseteq X: \tau \in \mathbb{R}, \omega \in \Omega\}$ of nonempty subsets of $X$ is invariant under $\Phi$ if $\Phi(t, \tau, \omega, B(\tau, \omega))=B\left(t+\tau, \theta_{t} \omega\right)$ for all $t \geq 0, \tau \in \mathbb{R}, \omega \in \Omega$.

Definition 2.3. Let $B=\{B(\tau, \omega) \subseteq X: \tau \in \mathbb{R}, \omega \in \Omega\}$ be a family of nonempty subsets of $X$. For every $\tau \in \mathbb{R}, \omega \in \Omega$,

$$
\omega(B)=\omega(B, \tau, \omega)=\bigcap_{r \geq 0} \overline{\bigcup_{t \geq r} \Phi\left(t, \tau-t, \theta_{-t} \omega, B\left(\tau-t, \theta_{-t} \omega\right)\right)}
$$

is called the $\omega$-limit set of $B$.

Definition 2.4. A family $D=\{D(\tau, \omega): \tau \in \mathbb{R}, \omega \in \Omega\}$ of nonempty bounded subsets of $X$ is said to be tempered with respect to $\left(\theta_{t}\right)_{t \in \mathbb{R}}$ if for every $\gamma>0, \lim _{t \rightarrow \infty} e^{-\gamma|t|} \| D(\tau+$ $\left.t, \theta_{t} \omega\right) \|_{X}=0$ for a.e. $\omega \in \Omega$, where $\|D\|_{X}=\sup _{x \in D}\|x\|$.

In the following, let $\mathcal{D}=\mathcal{D}(X)$ be the collection of all tempered families of nonempty subsets of $X$.

Definition 2.5. A family $K=\{K(\tau, \omega): \tau \in \mathbb{R}, \omega \in \Omega\} \in \mathcal{D}$ of nonempty subsets of $X$ is called a measurable $\mathcal{D}$-pullback attracting (or absorbing) set for $\Phi$ if

(i) $K$ is measurable with respect to the $\mathbb{P}$-completion of $\mathcal{F}$ in $\Omega$; 
(ii) for all $\tau \in \mathbb{R}, \omega \in \Omega$ and for every $B \in \mathcal{D}, \lim _{t \rightarrow+\infty} d\left(\Phi\left(t, \tau-t, \theta_{-t} \omega\right), B(\tau-\right.$ $\left.\left.t, \theta_{-t} \omega\right), K(\tau, \omega)\right)=0$, where $d(\cdot, \cdot)$ denotes the Hausdorff semi-distance between two subsets of $X$ (or there exists $T(B, \tau, \omega)>0$ such that $\Phi\left(t, \tau-t, \theta_{-t} \omega, B(\tau-\right.$ $\left.\left.\left.t, \theta_{-t} \omega\right)\right) \subseteq K(\tau, \omega), \forall t \geq T(B, \tau, \omega)>0\right)$.

Definition 2.6. A family $A=\{A(\tau, \omega): \tau \in \mathbb{R}, \omega \in \Omega\} \in \mathcal{D}$ is called a $\mathcal{D}$-pullback random attractor associated to the $\operatorname{RDS} \Phi$ if

(i) $A(\tau, \omega)$ is measurable in $\omega$ with respect to $\mathcal{F}$ and compact in $X$ for all $\tau \in \mathbb{R}, \omega \in \Omega$;

(ii) $A$ is invariant, i.e., $\Phi(t, \tau, \omega, A(\tau, \omega))=A\left(t+\tau, \theta_{t} \omega\right)$, for every $\tau \in \mathbb{R}, \omega \in \Omega, \forall t \geq 0$;

(iii) $A$ attracts every every member of $\mathcal{D}$, i.e., for every $B=\{B(\tau, \omega): \tau \in \mathbb{R}, \omega \in \Omega\} \in$ $\mathcal{D}$, and for every $\tau \in \mathbb{R}, \omega \in \Omega$,

$$
\lim _{t \rightarrow \infty} d\left(\Phi\left(t, \tau-t, \theta_{-t} \omega, B\left(\tau-t, \theta_{-t} \omega\right)\right), A(\tau, \omega)\right)=0 .
$$

Definition 2.7. A mapping $\psi: \mathbb{R} \times \mathbb{R} \times \Omega \rightarrow X$ is called a complete orbit of $\Phi$ if for every $t \geq 0, \tau, s \in \mathbb{R}$ and $\omega \in \Omega$, then $\Phi\left(t, \tau+s, \theta_{s} \omega, \psi(s, \tau, \omega)\right)=\psi(t+s, \tau, \omega)$. In addition, $\psi$ is called a $\mathcal{D}$-complete orbit of $\Phi$ if there exists $D=\{D(\tau, \omega): \tau \in \mathbb{R}, \omega \in \Omega\} \in \mathcal{D}$ such that $\psi(t, \tau, \omega)$ belongs to $D\left(t+\tau, \theta_{t} \omega\right)$ for every $t \in \mathbb{R}, \tau \in \mathbb{R}$ and $\omega \in \Omega$.

Theorem 2.8 (Existence of a random attractor). [20] Let $\Phi$ be a continuous $R D S$ on $X$ over $\mathbb{R}$ and $\left(\Omega, \mathcal{F}, \mathbb{P},\left(\theta_{t}\right)_{t \in \mathbb{R}}\right)$. Suppose that $\Phi$ has a compact measurable $\mathcal{D}$-pullback attracting set $K$ in $\mathcal{D}$, then $\Phi$ has a unique $\mathcal{D}$-pullback random attractor $\mathcal{A}$ in $\mathcal{D}$ given by the following: for every $\tau \in \mathbb{R}, \omega \in \Omega$,

$$
\begin{aligned}
\mathcal{A}(\tau, \omega) & =\bigcap_{r \geq 0} \overline{\bigcup_{t \geq r} \Phi\left(t, \tau-t, \theta_{-t} \omega, K\left(\tau-t, \theta_{-t} \omega\right)\right)} \\
& =\{\psi(0, \tau, \omega): \psi(\cdot, \tau, \omega) \text { is a } \mathcal{D} \text {-complete orbit of } \Phi\} .
\end{aligned}
$$

Theorem 2.9 (Upper semicontinuity of random attractors). 19 Let $I$ be an interval of $\mathbb{R}$ and given $a \in I$. Let $\left\{\Phi^{a}(t, \tau, \omega)\right\}_{a \in I}$ be a family of continuous $R D S$ on $X$ over $\mathbb{R}$ and $\left(\Omega, \mathcal{F}, \mathbb{P},\left(\theta_{t}\right)_{t \in \mathbb{R}}\right)$. Given $a_{0} \in I, \Phi^{a_{0}}(t, \tau)$ is a continuous process over $\mathbb{R}$ independent of $\omega \in \Omega$. Suppose that

(i) $\Phi^{a_{0}}(t, \tau)$ has a pullback attractor $\left\{\mathcal{A}^{a_{0}}(\tau)\right\}_{\tau \in \mathbb{R}}$ with properties: (a) $\mathcal{A}^{a_{0}}(\tau)$ is a compact for $\tau \in \mathbb{R}$; (b) $\Phi^{a_{0}}(t, \tau) \mathcal{A}^{a_{0}}(\tau)=\mathcal{A}^{a_{0}}(t)$ for $t \geq \tau$; (c) for any bounded set $B \subset X, \lim _{t \rightarrow \infty} d\left(\Phi^{a_{0}}(\tau, \tau-t) B, \mathcal{A}^{a_{0}}(\tau)\right)=0 ;$

(ii) $\Phi^{a_{0}}(t, \tau)$ has a uniform pullback absorbing set $B^{a_{0}}=\left\{x \in X:\|x\|_{X} \leq R^{a_{0}}\right\} \subset X$ and for each $a \in I, \Phi^{a}$ has a $\mathcal{D}(X)$-pullback random attractor $\mathcal{A}^{a}(\tau, \omega) \in \mathcal{D}(X)$ and a $\mathcal{D}(X)$-pullback random absorbing set $K^{a}(\tau, \omega) \in \mathcal{D}(X)$ such that for all $\tau \in \mathbb{R}$, $\omega \in \Omega, \limsup _{a \rightarrow a_{0}}\left\|K^{a}(\tau, \omega)\right\|_{X} \leq R^{a_{0}} ;$ 
(iii) for every $\tau \in \mathbb{R}, \omega \in \Omega, \bigcup_{a \in I} \mathcal{A}^{a}(\tau, \omega)$ is precompact in $X$;

(iv) for every $t \in \mathbb{R}^{+}, \tau \in \mathbb{R}, \omega \in \Omega, a_{n} \in I$ with $a_{n} \rightarrow a$ and $x_{n}, x \in X$ with $x_{n} \rightarrow x$, $\lim _{n \rightarrow \infty} \Phi^{a_{n}}\left(t, \tau-t, \theta_{-t} \omega, x_{n}\right)=\Phi^{a_{0}}(t, \tau-t, x)$.

Then for every $\tau \in \mathbb{R}, \omega \in \Omega, d\left(\mathcal{A}^{a}(\tau, \omega), \mathcal{A}^{a_{0}}(\tau)\right) \rightarrow 0$ as $a \rightarrow a_{0}$.

\section{Existence and uniqueness of solutions}

In the sequel, we always assume that the conditions $1.6-1.8$ hold, the ergodic metric dynamical system $\left(\Omega, \mathcal{F}, \mathbb{P},\left(\theta_{t}\right)_{t \in \mathbb{R}}\right)$ is defined as in Section 2 .

We consider the $H=L^{2}(U), V=H^{2}(U) \cap H_{0}^{1}(U)$ with the usual inner products and norms, respectively,

$$
\begin{aligned}
(u, v) & =\int_{U} u v d x, \quad\|u\|^{2}=(u, u), \quad \forall u, v \in H \\
((u, v)) & =\int_{U} \triangle u \triangle v d x, \quad\|u\|_{2}^{2}=((u, u)), \quad \forall u, v \in V .
\end{aligned}
$$

Let $E=V \times H$, and endow space $E$ with the scalar product and the norm:

$$
\left(y_{1}, y_{2}\right)_{E}=\left(\left(u_{1}, u_{2}\right)\right)+\left(v_{1}, v_{2}\right), \quad\|y\|_{E}^{2}=(y, y)_{E}
$$

for all $y_{i}=\left(u_{i}, v_{i}\right)^{T}, y=(u, v)^{T} \in E$, here $T$ denotes the transposition.

Without loss of generality, we define $\mathcal{H}^{r}=D\left(A^{r / 4}\right)$ for $r \in \mathbb{R}$, which turns out to be a Hilbert space with the inner product $(u, v)_{r}=\left(A^{r / 4} u, A^{r / 4} v\right)$. We also consider by $\|\cdot\|_{r}=\left\|A^{r / 4} u\right\|$ the norm on $\mathcal{H}^{r}$ induced by the above inner product. It is well-known that $D\left(A^{0}\right)=H, D\left(A^{1 / 2}\right)=V, D(A)=\left\{u \in H^{4}(U): u, \triangle u \in H_{0}^{1}(U)\right\}$ with $A=\triangle^{2}$, $A^{1 / 2}=-\triangle$. Thus, by the compact embeddings $\mathcal{H}^{r+1} \hookrightarrow \mathcal{H}^{r}$ along with the the generalized Poincré inequality, then

$$
\|u\|_{r+1}^{4} \geq \lambda_{1}\|u\|_{r}^{4}, \quad \forall u \in \mathcal{H}^{r+1}
$$

where $\lambda_{1}>0$ is the first eigenvalue of $A$.

We define

$$
\varepsilon=\frac{\lambda_{1}^{2}}{4 \lambda_{1}^{2}+3 \lambda_{1}+4} .
$$

To convert the stochastic equation (1.3) into a deterministic one with random parameters but without noise terms, we introduce an Ornstein-Uhlenbeck process driven by the Brownian motion, which satisfies the Itô stochastic differential equation

$$
d z+\varepsilon z d t=d W(t)
$$


and is given by

$$
z(t)=z\left(\theta_{t} \omega\right):=-\varepsilon \int_{-\infty}^{0} e^{\varepsilon s}\left(\theta_{t} \omega\right)(s) d s, \quad t \in \mathbb{R}, \omega \in \Omega .
$$

By 2, 4, we have the identity $W(t, \omega)=\omega(t)$, and there is a $\theta_{t}$-invariant set $\omega \in \widetilde{\Omega}$ of full $\mathbb{P}$ measure such that for every $\omega \in \widetilde{\Omega}, t \rightarrow z\left(\theta_{t} \omega\right)$ is continuous in $t$ and

$$
\lim _{t \rightarrow+\infty} e^{-\gamma t}\left|z\left(\theta_{-t} \omega\right)\right|=0, \quad \forall \gamma>0, \omega \in \widetilde{\Omega}
$$

the random variable $|z(\omega)|$ is tempered. For convenience, we shall simply write $\Omega$ as $\widetilde{\Omega}$.

Let $u(t, \tau, \omega, x)=u, v(t, \tau, \omega, x)=u_{t}(t, \tau, \omega, x)+\varepsilon u(t, \tau, \omega, x)-a q(x) z\left(\theta_{t} \omega\right)$, we get the new random partial differential equation (RPDE) as follows:

$$
\begin{aligned}
& \frac{d u}{d t}= v-\varepsilon u+a q(x) z\left(\theta_{t} \omega\right) \\
& \frac{d v}{d t}=-(1-\varepsilon) A u-\varepsilon^{2} u-A v+\varepsilon v+\left(p-\|u\|_{1}^{2}\right) A^{1 / 2} u-k u^{+} \\
& \quad-f(u, x)+g(x, t)-a A q(x) z\left(\theta_{t} \omega\right)+2 a \varepsilon q(x) z\left(\theta_{t} \omega\right) \\
& u(t, \tau, x)=u_{\tau}(t, \tau, x), \quad v(t, \tau, x)=u_{1, \tau}(t, \tau, x)+\varepsilon u_{\tau}(t, \tau, x)-a q(x) z\left(\theta_{t} \omega\right) .
\end{aligned}
$$

In contrast to the stochastic differential equation $(1.3)$, no stochastic differential appears here. Set

$$
\begin{aligned}
\varphi=\left(\begin{array}{l}
u \\
v
\end{array}\right), \quad L=\left(\begin{array}{cc}
\varepsilon I & -I \\
(1-\varepsilon) A+\varepsilon^{2} I & A-\varepsilon I
\end{array}\right), \\
F\left(\varphi, \theta_{t} \omega, t\right) \\
=\left(\begin{array}{c}
a q(x) z\left(\theta_{t} \omega\right) \\
\left(p-\|u\|_{1}^{2}\right) A^{1 / 2} u-k u^{+}-f(u, x)+g(x, t)-a A q(x) z\left(\theta_{t} \omega\right)+2 a \varepsilon q(x) z\left(\theta_{t} \omega\right)
\end{array}\right),
\end{aligned}
$$

then 1.3 can be reinterpreted as

$$
\dot{\varphi}+L \varphi=F\left(\varphi, \theta_{t} \omega, t\right), \quad \varphi_{\tau}(\omega)=\left(u_{\tau}, u_{1, \tau}+\varepsilon u_{\tau}-a q(x) z\left(\theta_{t} \omega\right)\right)^{T}
$$

By [18] we have the fact that $L$ is the infinitesimal generators of $C_{0}$-semigroup $e^{L t}$ on $E$. It is easy to prove that the functions $F\left(\cdot, \theta_{t} \omega, t\right): E \mapsto E$ is locally Lipschitz continuous with respect to $\varphi$ and bounded for every $\omega \in \Omega$. Thus, by the classical semigroup theory of existence and uniqueness of solutions of evolution differential equations in [18], we have the following theorems. 
Theorem 3.1. If (1.6)-1.8) hold, then for any $\tau \in \mathbb{R}, \omega \in \Omega$ and $\varphi_{\tau}=\left(u_{\tau}, v_{\tau}\right)^{T} \in E$, the $R P D E(3.3)$ has a unique solution $\varphi\left(\cdot, \tau, \omega, \varphi_{\tau}\right) \in C([\tau, \tau+T) ; E)$ with $\varphi\left(\tau, \tau, \omega, \varphi_{\tau}\right) \in E$ in mild sense, i.e.,

$$
\varphi\left(t, \tau, \omega, \varphi_{\tau}\right)=e^{-L(t-\tau)} \varphi_{\tau}(\omega)+\int_{\tau}^{t} e^{-L(t-s)} F\left(\varphi\left(r, \tau, \omega, \varphi_{\tau}\right), \theta_{r} \omega, r\right) d r, \quad \forall t \geq \tau .
$$

Moreover, $\varphi\left(t, \tau, \omega, \varphi_{\tau}\right)$ is continuous in $\varphi_{\tau}$ and measurable in $\omega$.

Theorem 3.2. If (1.6)-(1.8) hold, then for $t \in \mathbb{R}^{+}, \tau \in \mathbb{R}$ and $\omega \in \Omega$, the solution $\varphi\left(t, \tau, \omega, \varphi_{\tau}\right)$ of system 3.3 generates a continuous random dynamical system over $\mathbb{R}$ and $\left(\Omega, \mathcal{F}, \mathbb{P},\left(\theta_{t}\right)_{t \in \mathbb{R}}\right):$

$$
\Phi: \mathbb{R}^{+} \times \mathbb{R} \times \Omega \times E \rightarrow E, \quad\left(t, \tau, \omega, \varphi_{\tau}\right) \mapsto \Phi\left(t, \tau, \omega, \varphi_{\tau}\right)
$$

by

$$
\begin{aligned}
& \Phi\left(t, \tau, \omega, \varphi_{\tau}(\omega)\right)=\varphi\left(t+\tau, \tau, \theta_{-\tau} \omega, \varphi_{\tau}\left(\theta_{-\tau} \omega\right)\right) \\
= & \left(\begin{array}{c}
u\left(t+\tau, \tau, \theta_{-\tau} \omega, \varphi_{\tau}\left(\theta_{-\tau} \omega\right)\right) \\
u_{t}\left(t+\tau, \tau, \theta_{-\tau} \omega, \varphi_{\tau}\left(\theta_{-\tau} \omega\right)\right)+\varepsilon u\left(t+\tau, \tau, \theta_{-\tau} \omega, \varphi_{\tau}\left(\theta_{-\tau} \omega\right)\right)-a q(x) z\left(\theta_{t} \omega\right)
\end{array}\right),
\end{aligned}
$$

where

$$
\begin{aligned}
\Phi\left(0, \tau, \omega, \varphi_{\tau}(\omega)\right) & =\left(\begin{array}{c}
u\left(\tau, \tau, \theta_{-\tau} \omega, \varphi_{\tau}\left(\theta_{-\tau} \omega\right)\right) \\
u_{t}\left(\tau, \tau, \theta_{-\tau} \omega, \varphi_{\tau}\left(\theta_{-\tau} \omega\right)\right)+\varepsilon u\left(\tau, \tau, \theta_{-\tau} \omega, \varphi_{\tau}\left(\theta_{-\tau} \omega\right)\right)-a q(x) z(\omega)
\end{array}\right) \\
& =\varphi_{\tau}\left(\theta_{-\tau} \omega\right)
\end{aligned}
$$

and

$$
\Phi\left(t, \tau-t, \theta_{-t} \omega, \varphi_{\tau-t}\left(\theta_{-\tau} \omega\right)\right)=\varphi\left(\tau, \tau-t, \theta_{-\tau} \omega, \varphi_{\tau-t}\left(\theta_{-\tau} \omega\right)\right) .
$$

To consider the conjugation of the solution of the stochastic partial differential equation (1.3) and the random partial differential equation (3.3), we defined the homeomorphism

$$
R\left(\theta_{t} \omega\right):\left(y_{1}, y_{2}\right)^{T} \rightarrow\left(y_{1}, y_{2}-\varepsilon y_{1}+a q(x) z\left(\theta_{t} \omega\right)\right)^{T} .
$$

It is easy to find that the transformation

$$
\widetilde{\Phi}\left(t, \tau, \omega, Z_{\tau}\right)=R\left(\theta_{t} \omega\right) \Phi\left(t, \tau, \omega, \varphi_{\tau}\right) R^{-1}\left(\theta_{t} \omega\right): Z_{\tau} \rightarrow Z\left(t+\tau, \tau, \theta_{-\tau} \omega, Z_{\tau}\right)
$$

determines a random dynamical systems corresponding to the equation (1.3). Similarly, the transformation

$$
\widehat{\Phi}\left(t, \tau, \omega, \psi_{\tau}\right)=T_{\varepsilon} \widetilde{\Phi}\left(t, \tau, \omega, Z_{\tau}\right) T_{-\varepsilon}: \psi_{\tau} \rightarrow \psi\left(t+\tau, \tau, \theta_{-\tau} \omega, \psi_{\tau}\right)
$$

is also a random dynamical systems corresponding to the equation 3.3 . Therefore, $\Phi, \widetilde{\Phi}$ and $\widehat{\Phi}$ are equivalent to each other in dynamics. 


\section{Existence of random attractor}

In this section, we first show the existence of a pullback absorbing set for $\Phi$ in $\mathcal{D}(E)$, then we prove the cocycle associated with non-autonomous a stochastic Kirchhoff type suspension bridge equation with strong damping (1.3) has a random attractor in $\left(H^{2}(U) \cap\right.$ $\left.H_{0}^{1}(U)\right) \times L^{2}(U)$, which is a tempered random set in the space $H^{3}(U) \times\left(H^{2}(U) \cap H_{0}^{1}(U)\right)$.

Lemma 4.1. For any $\varphi=(u, v)^{T} \in E$, there holds

$$
(L \varphi, \varphi)_{E} \geq \frac{\varepsilon}{2}\|\varphi\|_{E}^{2}+\frac{\varepsilon}{4}\|u\|_{2}^{2}+\frac{\lambda_{1}}{2}\|v\|^{2} .
$$

Proof. Note that $L \varphi=\left(\varepsilon u-v,(1-\varepsilon) A u+\varepsilon^{2} u+A v-\varepsilon v\right)^{T}$, which implies by the Young inequality and Poincaré inequality that

$$
\begin{aligned}
(L \varphi, \varphi)_{E} & =\varepsilon\|u\|_{2}^{2}-\varepsilon\left(A^{1 / 2} u, A^{1 / 2} v\right)+\varepsilon^{2}(u, v)+\left\|A^{1 / 2} v\right\|^{2}-\varepsilon\|v\|^{2} \\
& \geq \varepsilon\|u\|_{2}^{2}-\frac{\varepsilon}{8}\|u\|_{2}^{2}-2 \varepsilon\left\|A^{1 / 2} v\right\|^{2}-\frac{\varepsilon}{8}\|u\|_{2}^{2}-\frac{2 \varepsilon^{3}}{\lambda_{1}}\|v\|^{2}+\left\|A^{1 / 2} v\right\|^{2}-\varepsilon\|v\|^{2} \\
& \geq \frac{\varepsilon}{2}\|\varphi\|_{E}^{2}+\frac{\varepsilon}{4}\|u\|_{2}^{2}+(1-2 \varepsilon) \lambda_{1}\|v\|^{2}-\left(\frac{2 \varepsilon}{\lambda_{1}}+\frac{3 \varepsilon}{2}\right)\|v\|^{2} \\
& =\frac{\varepsilon}{2}\|\varphi\|_{E}^{2}+\frac{\varepsilon}{4}\|u\|_{2}^{2}+\frac{\lambda_{1}}{2}\|v\|^{2},
\end{aligned}
$$

for the fact that we used $\varepsilon=\lambda_{1}^{2} /\left(4 \lambda_{1}^{2}+3 \lambda_{1}+4\right)$ in the last inequality.

Lemma 4.2. Let $1.6-1.8$ hold, $q(x) \in H^{5}(U) \cap H_{0}^{3}(U)$. For any $\tau \in \mathbb{R}, \omega \in \Omega$, there exists a closed tempered measurable absorbing ball $B_{0}(\omega)=\left\{\varphi \in E:\|\varphi\|_{E} \leq\right.$ $\left.r_{0}(\omega)\right\}=B_{E}\left(0, r_{0}(\omega)\right) \in \mathcal{D}(E)$ of $E$, centered at 0 of radius $r_{0}(\omega)>0$ such that for any set $B \in \mathcal{D}(E)$, there exists $T(\tau, \omega, B) \geq 0$ such that the mild solution $\varphi(\tau, \tau-$ $\left.t, \theta_{-\tau} \omega, \varphi_{\tau-t}\left(\theta_{-\tau} \omega\right)\right) \in E$ of (3.3) with $\varphi_{\tau-t}\left(\theta_{-\tau} \omega\right) \in B\left(\tau-t, \theta_{-t} \omega\right)$ satisfies

$$
\begin{aligned}
\left\|\varphi\left(\tau, \tau-t, \theta_{-\tau} \omega, \varphi_{\tau-t}\left(\theta_{-\tau} \omega\right)\right)\right\|_{E}^{2}= & \left\|u\left(\tau, \tau-t, \theta_{-\tau} \omega, \varphi_{\tau-t}\left(\theta_{-\tau} \omega\right)\right)\right\|_{2}^{2} \\
& +\left\|v\left(\tau, \tau-t, \theta_{-\tau} \omega, \varphi_{\tau-t}\left(\theta_{-\tau} \omega\right)\right)\right\|^{2} \\
\leq & r_{0}^{2}(\omega), \quad \forall t \geq T(\tau, \omega, B),
\end{aligned}
$$

that is,

$$
\Phi\left(t, \tau-t, \theta_{-t} \omega, B\left(\tau-t, \theta_{-t} \omega\right)\right) \subseteq B_{0}(\omega), \quad \forall t \geq T(\tau, \omega, B)
$$

Proof. For any $\tau \in \mathbb{R}, \omega \in \Omega, t \geq 0$, let $\varphi(r)=\varphi\left(r, \tau-t, \theta_{-\tau} \omega, \varphi_{\tau-t}\left(\theta_{-\tau} \omega\right)\right)=$ $(u(r), v(r))^{T} \in E(r \geq \tau-t)$ be a mild solution of $(3.3)$ with initial value $\varphi_{\tau-t}\left(\theta_{-\tau} \omega\right)=$ $\left(u_{\tau-t}, u_{1, \tau-t}+\varepsilon u_{\tau-t}-a q(x) z\left(\theta_{-t} \omega\right)\right)^{T} \in E$. Taking the inner product $(\cdot, \cdot)_{E}$ of (3.3) with $\varphi(r)$, we have that for $r \geq \tau-t$,

$$
\frac{1}{2} \frac{d}{d t}\|\varphi(r)\|_{E}^{2}+(L \varphi, \varphi)_{E}=\left(F\left(\varphi, \theta_{r-\tau} \omega, t\right), \varphi\right)_{E},
$$


where

$$
\begin{aligned}
\left(F\left(\varphi, \theta_{r-\tau} \omega, t\right), \varphi\right)_{E}= & \left(\left(a q(x) z\left(\theta_{r-\tau} \omega\right), u\right)\right)-\left(\|u\|_{1}^{2}-p\right)\left(A^{1 / 2} u, v\right)-k\left(u^{+}, v\right) \\
& -(f(u, x), v)+(g(x, r), v)+\left(2 a \varepsilon q(x) z\left(\theta_{r-\tau} \omega\right), v\right) \\
& -\left(a A \varepsilon q(x) z\left(\theta_{r-\tau} \omega\right), v\right) .
\end{aligned}
$$

To estimate the terms in 4.3 one by one as follows,

$$
\begin{gathered}
\left(\left(a q(x) z\left(\theta_{r-\tau} \omega\right), u\right)\right) \leq \frac{\varepsilon}{4}\|u\|_{2}^{2}+\frac{a^{2}\|q\|_{2}^{2}}{\varepsilon}\left|z\left(\theta_{r-\tau} \omega\right)\right|^{2}, \\
-k\left(u^{+}, v\right)=-\frac{1}{2} \frac{d}{d r} k\left\|u^{+}\right\|^{2}-\varepsilon k\left\|u^{+}\right\|^{2}+k\left(u^{+}, a q(x) z\left(\theta_{r-\tau} \omega\right)\right) \\
\leq-\frac{1}{2} \frac{d}{d r} k\left\|u^{+}\right\|^{2}-\frac{\varepsilon k}{2}\left\|u^{+}\right\|^{2}+\frac{k a^{2}\|q\|^{2}}{2 \varepsilon}\left|z\left(\theta_{r-\tau} \omega\right)\right|^{2}, \\
\left(2 a \varepsilon q(x) z\left(\theta_{r-\tau} \omega\right), v\right) \leq \frac{8 a^{2} \varepsilon^{2}\|q\|^{2}}{\lambda_{1}}\left|z\left(\theta_{r-\tau} \omega\right)\right|^{2}+\frac{\lambda_{1}}{8}\|v\|^{2}, \\
\left|-\left(a A \varepsilon q(x) z\left(\theta_{r-\tau} \omega\right), v\right)\right| \leq \frac{2\|q\|_{4}^{2}}{\lambda_{1}}\left|z\left(\theta_{r-\tau} \omega\right)\right|^{2}+\frac{\lambda_{1}}{8}\|v\|^{2}, \\
(g(x, r), v) \leq \frac{1}{\lambda_{1}}\|g\|^{2}+\frac{\lambda_{1}}{4}\|v\|^{2},
\end{gathered}
$$

where $\|g\|^{2}=\sup _{r \in \mathbb{R}}\|g(\cdot, r)\|^{2}<\infty$. By a standard computation, we have

$$
\begin{aligned}
-\left(\|u\|_{1}^{2}-p\right)\left(A^{1 / 2} u, v\right)= & -\frac{1}{4} \frac{d}{d t}\left(\|u\|_{1}^{2}-p\right)^{2}-\frac{\varepsilon}{2}\left(\|u\|_{1}^{2}-p\right)^{2}-\frac{\varepsilon}{2}\|u\|_{1}^{4}+\frac{\varepsilon p^{2}}{2} \\
& +\left(\|u\|_{1}^{2}-p\right)\left(A^{1 / 2} u, a q(x) z\left(\theta_{r-\tau} \omega\right)\right) \\
\leq & -\frac{1}{4} \frac{d}{d t}\left(\|u\|_{1}^{2}-p\right)^{2}-\frac{\varepsilon}{2}\left(\|u\|_{1}^{2}-p\right)^{2}-\frac{\varepsilon}{2}\|u\|_{1}^{4} \\
& +\frac{\varepsilon p^{2}}{2}+\frac{\varepsilon}{4}\left(\|u\|_{1}^{2}-p\right)^{2}+\frac{\varepsilon}{2}\|u\|_{1}^{4}+\frac{a^{2}\|q\|_{1}^{4}}{2 \varepsilon^{3}}\left|z\left(\theta_{r-\tau} \omega\right)\right|^{4} \\
= & -\frac{1}{4} \frac{d}{d t}\left(\|u\|_{1}^{2}-p\right)^{2}-\frac{\varepsilon}{4}\left(\|u\|_{1}^{2}-p\right)^{2}+\frac{\varepsilon p^{2}}{2} \\
& +\frac{a^{2}\|q\|_{1}^{4}}{2 \varepsilon^{3}}\left|z\left(\theta_{r-\tau} \omega\right)\right|^{4}
\end{aligned}
$$

By (1.6), 1.7), Hölder inequality and the Sobolev embedding theorem, we obtain

$$
\begin{aligned}
& \left(f(u, x), a q(x) z\left(\theta_{r-\tau} \omega\right)\right) \\
\leq & \int_{U}\left(C_{0}|u|^{\gamma}+\left|\phi_{1}(x)\right|\right)\left|a q(x) z\left(\theta_{r-\tau} \omega\right)\right| d x \\
\leq & |a|\left\|\phi_{1}\right\|\|q\|\left|z\left(\theta_{r-\tau} \omega\right)\right|+|a| C_{0}\left(\int_{U}|u|^{\gamma+1} d x\right)^{\gamma /(\gamma+1)}\|q\|_{L^{\gamma+1}}\left|z\left(\theta_{r-\tau} \omega\right)\right| \\
\leq & \frac{1}{2} a^{2}\left\|\phi_{1}\right\|^{2}+\frac{a^{2}}{2}\|q\|^{2}\left|z\left(\theta_{r-\tau} \omega\right)\right|^{2}
\end{aligned}
$$




$$
\begin{aligned}
& +|a| C_{0} C_{1}^{-\gamma /(\gamma+1)}\left(\int_{U}\left(F(x, u)+\phi_{2}(x)\right) d x\right)^{\gamma /(\gamma+1)}\|q\|_{L^{\gamma+1}}\left|z\left(\theta_{r-\tau} \omega\right)\right| \\
\leq & \frac{1}{2} a^{2}\left\|\phi_{1}\right\|^{2}+\frac{a^{2}}{2}\|q\|^{2}\left|z\left(\theta_{r-\tau} \omega\right)\right|^{2}+\frac{\varepsilon|a| C_{0} C_{1}^{-1}}{2} \int_{U} F(x, u) d x \\
& +\frac{|a| C_{0}}{2 \varepsilon}\|q\|_{2}^{\gamma+1}\left|z\left(\theta_{r-\tau} \omega\right)\right|^{\gamma+1}+\frac{\varepsilon|a| C_{0} C_{1}^{-1}}{2}\left\|\phi_{2}\right\|_{L^{1}} .
\end{aligned}
$$

Combining the above inequality with 1.8 implies that

$$
\begin{aligned}
-(f(u, x), v)= & -\left(f(u, x), u_{t}+\varepsilon u-a q(x) z\left(\theta_{r-\tau} \omega\right)\right) \\
\leq & -\frac{d}{d t} \int_{U} F(u, x) d x-\varepsilon C_{2} \int_{U} F(x, u) d x+\varepsilon \int_{U} \phi_{3}(x) d x \\
& +\left(f(u, x), a q(x) z\left(\theta_{r-\tau} \omega\right)\right) \\
\leq & -\frac{d}{d t} \int_{U} F(u, x) d x-\frac{\varepsilon\left(2 C_{2}-|a| C_{0} C_{1}^{-1}\right)}{2} \int_{U} F(u, x) d x \\
& +\frac{1}{2}\|q\|^{2}\left|z\left(\theta_{r-\tau} \omega\right)\right|^{2}+\frac{|a| C_{0}}{2 \varepsilon}\|q\|_{2}^{\gamma+1}\left|z\left(\theta_{r-\tau} \omega\right)\right|^{\gamma+1}+\frac{1}{2} a^{2}\left\|\phi_{1}\right\|^{2} \\
& +\frac{\varepsilon|a| C_{0} C_{1}^{-1}}{2}\left\|\phi_{2}\right\|_{L^{1}}+\varepsilon\left\|\phi_{3}\right\|_{L^{1}} .
\end{aligned}
$$

By putting 4.3 -4.11) into 4.2, we have that for $r \geq \tau-t$,

$$
\begin{aligned}
& \quad \frac{d}{d t}\left(\|\varphi(r)\|_{E}^{2}+\frac{1}{2}\left(\|u\|_{1}^{2}-p\right)^{2}+k\left\|u^{+}\right\|^{2}+2 \int_{U} F(u, x) d x\right) \\
& +\varepsilon\left(\|\varphi(r)\|_{E}^{2}+\frac{1}{2}\left(\|u\|_{1}^{2}-p\right)^{2}+k\left\|u^{+}\right\|^{2}+\left(2 C_{2}-|a| C_{0} C_{1}^{-1}\right) \int_{U} F(u, x) d x\right) \\
& \leq \frac{2 a^{2}\|q\|_{2}^{2}}{\varepsilon}\left|z\left(\theta_{r-\tau} \omega\right)\right|^{2}+\frac{k a^{2}\|q\|^{2}}{\varepsilon}\left|z\left(\theta_{r-\tau} \omega\right)\right|^{2}+\frac{16 a^{2} \varepsilon^{2}\|q\|^{2}}{\lambda_{1}}\left|z\left(\theta_{r-\tau} \omega\right)\right|^{2} \\
& +\frac{4\|q\|_{4}^{2}}{\lambda_{1}}\left|z\left(\theta_{r-\tau} \omega\right)\right|^{2}+\frac{a^{2}\|q\|_{1}^{4}}{\varepsilon^{3}}\left|z\left(\theta_{r-\tau} \omega\right)\right|^{4}+a^{2}\|q\|^{2}\left|z\left(\theta_{r-\tau} \omega\right)\right|^{2} \\
& +\frac{|a| C_{0}}{\varepsilon}\|q\|_{2}^{\gamma+1}\left|z\left(\theta_{r-\tau} \omega\right)\right|^{\gamma+1}+a^{2}\left\|\phi_{1}\right\|^{2}+\varepsilon|a| C_{0} C_{1}^{-1}\left\|\phi_{2}\right\|_{L^{1}} \\
& +2 \varepsilon\left\|\phi_{3}\right\|_{L^{1}}+\frac{2}{\lambda_{1}}\|g\|^{2}+\varepsilon p^{2} .
\end{aligned}
$$

Choosing $\varepsilon_{1}=\min \left\{\varepsilon, \varepsilon\left(2 C_{2}-|a| C_{0} C_{1}^{-1}\right) / 2\right\}\left(C_{2}>|a| C_{0} C_{1}^{-1} / 2\right)$, and set

$$
\begin{aligned}
\chi(r) & =\|\varphi(r)\|_{E}^{2}+\frac{1}{2}\left(\|u\|_{1}^{2}-p\right)^{2}+k\left\|u^{+}\right\|^{2}+2 \int_{U} F(u, x) d x \\
& \geq\|\varphi(r)\|_{E}^{2}-2\left\|\phi_{2}\right\|_{L^{1}},
\end{aligned}
$$




$$
\begin{aligned}
m_{1}\left(\theta_{r-\tau} \omega\right)= & \frac{2 a^{2}\|q\|_{2}^{2}}{\varepsilon}\left|z\left(\theta_{r-\tau} \omega\right)\right|^{2}+\frac{k a^{2}\|q\|^{2}}{\varepsilon}\left|z\left(\theta_{r-\tau} \omega\right)\right|^{2}+\frac{16 a^{2} \varepsilon^{2}\|q\|^{2}}{\lambda_{1}}\left|z\left(\theta_{r-\tau} \omega\right)\right|^{2} \\
& +\frac{4\|q\|_{4}^{2}}{\lambda_{1}}\left|z\left(\theta_{r-\tau} \omega\right)\right|^{2}+\frac{a^{2}\|q\|_{1}^{4}}{\varepsilon^{3}}\left|z\left(\theta_{r-\tau} \omega\right)\right|^{4}+a^{2}\|q\|^{2}\left|z\left(\theta_{r-\tau} \omega\right)\right|^{2} \\
& +\frac{|a| C_{0}}{\varepsilon}\|q\|_{2}^{\gamma+1}\left|z\left(\theta_{r-\tau} \omega\right)\right|^{\gamma+1}+a^{2}\left\|\phi_{1}\right\|^{2}+\varepsilon|a| C_{0} C_{1}^{-1}\left\|\phi_{2}\right\|_{L^{1}} \\
& +2 \varepsilon\left\|\phi_{3}\right\|_{L^{1}}+\frac{2}{\lambda_{1}}\|g\|^{2}+\varepsilon p^{2} .
\end{aligned}
$$

We can derive from 4.12 that

$$
\frac{d}{d t} \chi(r)+\varepsilon_{1} \chi(r) \leq m_{1}\left(\theta_{r-\tau} \omega\right) .
$$

By Gronwall lemma to 4.14) on $[\tau-t, r](r \geq \tau-t)$, we have that for $r \geq \tau-t$,

$$
\begin{aligned}
\chi\left(r, \tau-t, \theta_{-\tau} \omega, \varphi_{\tau-t}\left(\theta_{-\tau} \omega\right)\right) \leq & \chi\left(\tau-t, \tau-t, \theta_{-\tau} \omega, \varphi_{\tau-t}\left(\theta_{-\tau} \omega\right)\right) e^{-\varepsilon_{1}(r+t-\tau)} \\
& +\int_{\tau-t}^{r} m_{1}\left(\theta_{s-\tau} \omega\right) e^{-\varepsilon_{1}(r-s)} d s,
\end{aligned}
$$

where

$$
\begin{aligned}
& \chi\left(\tau-t, \tau-t, \theta_{-\tau} \omega, \varphi_{\tau-t}\left(\theta_{-\tau} \omega\right)\right) \\
= & \left\|\varphi(\tau-t)\left(\theta_{-\tau} \omega\right)\right\|_{E}^{2}+\frac{1}{2}\left(\left\|u_{\tau-t}\right\|_{1}^{2}-p\right)^{2}+k\left\|u_{\tau-t}^{+}\right\|^{2}+2 \int_{U} F(u(\tau-t), x) d x \\
\leq & \left\|\varphi(\tau-t)\left(\theta_{-\tau} \omega\right)\right\|_{E}^{2}+\frac{1}{2}\left(\left\|u_{\tau-t}\right\|_{1}^{2}-p\right)^{2}+k\left\|u_{\tau-t}^{+}\right\|^{2} \\
& +2 C_{3}\left(\left\|u_{\tau-t}\right\|^{2}+\left\|u_{\tau-t}\right\|_{\gamma+1}^{\gamma+1}+\left\|\phi_{1}\right\|^{2}+\left\|\phi_{3}\right\|_{L_{1}}\right) \\
\leq & \left\|\varphi(\tau-t)\left(\theta_{-\tau} \omega\right)\right\|_{E}^{2}+\frac{1}{2}\left(\left\|u_{\tau-t}\right\|_{1}^{2}-p\right)^{2}+k\left\|u_{\tau-t}^{+}\right\|^{2} \\
& +2 C_{3}\left(\left\|u_{\tau-t}\right\|^{2}+\left\|u_{\tau-t}\right\|_{2}^{\gamma+1}+\left\|\phi_{1}\right\|^{2}+\left\|\phi_{3}\right\|_{L_{1}}\right)
\end{aligned}
$$

and

$$
\begin{aligned}
& \int_{\tau-t}^{r} m_{1}\left(\theta_{s-\tau} \omega\right) e^{-\varepsilon_{1}(r-s)} d s \\
= & a^{2}\left(C_{4} \int_{\tau-t}^{r}\left|z\left(\theta_{s-\tau} \omega\right)\right|^{\gamma+1} e^{-\varepsilon_{1}(r-s)} d s+C_{5} \int_{\tau-t}^{r}\left|z\left(\theta_{s-\tau} \omega\right)\right|^{4} e^{-\varepsilon_{1}(r-s)} d s+C_{6}\right) .
\end{aligned}
$$

Thus, by 4.13 and 4.15, we have that for $r \geq \tau-t$,

$$
\begin{aligned}
& \left\|\varphi\left(r, \tau-t, \theta_{-\tau} \omega, \varphi_{\tau-t}\left(\theta_{-\tau} \omega\right)\right)\right\|_{E}^{2} \leq \chi(r)+2\left\|\phi_{2}\right\|_{L^{1}} \\
& \leq\left(\left\|\varphi(\tau-t)\left(\theta_{-\tau} \omega\right)\right\|_{E}^{2}+\frac{1}{2}\left(\left\|u_{\tau-t}\right\|_{1}^{2}-p\right)^{2}+k\left\|u_{\tau-t}^{+}\right\|^{2}\right. \\
& \left.\quad+2 C_{3}\left(\left\|u_{\tau-t}\right\|^{2}+\left\|u_{\tau-t}\right\|_{2}^{\gamma+1}+\left\|\phi_{1}\right\|^{2}+\left\|\phi_{3}\right\|_{L_{1}}\right)\right) e^{-\varepsilon_{1}(r+t-\tau)} \\
& \quad+\int_{\tau-t}^{r} m_{1}\left(\theta_{s-\tau} \omega\right) e^{-\varepsilon_{1}(r-s)} d s+2\left\|\phi_{2}\right\|_{L^{1}} .
\end{aligned}
$$


Therefore,

$$
\begin{aligned}
& \left\|\varphi\left(\tau, \tau-t, \theta_{-\tau} \omega, \varphi_{\tau-t}\left(\theta_{-\tau} \omega\right)\right)\right\|_{E}^{2} \\
\leq & \left\|\varphi(\tau-t)\left(\theta_{-\tau} \omega\right)\right\|_{E}^{2}+\frac{1}{2}\left(\left\|u_{\tau-t}\right\|_{1}^{2}-p\right)^{2}+k\left\|u_{\tau-t}^{+}\right\|^{2} \\
& +2 C_{3}\left(\left\|u_{\tau-t}\right\|^{2}+\left\|u_{\tau-t}\right\|_{2}^{\gamma+1}+\left\|\phi_{1}\right\|^{2}+\left\|\phi_{3}\right\|_{L_{1}}\right) e^{-\varepsilon_{1} t} \\
& +a^{2}\left(C_{4} \int_{-\infty}^{0}\left|z\left(\theta_{s} \omega\right)\right|^{\gamma+1} e^{\varepsilon_{1} s} d s+C_{5} \int_{-\infty}^{0}\left|z\left(\theta_{s} \omega\right)\right|^{4} e^{\varepsilon_{1} s} d s+\frac{2}{a^{2}}\left\|\phi_{2}\right\|_{L^{1}}+C_{6}\right) .
\end{aligned}
$$

For any set $B(\tau, \omega) \in \mathcal{D}(E)$, we obtain

$$
\varphi_{\tau-t}\left(\theta_{-\tau} \omega\right)=\left(u_{\tau-t}, u_{1, \tau-t}+\varepsilon u_{\tau-t}-a q(x) z\left(\theta_{-t} \omega\right)\right)^{T} \in B\left(\tau-t, \theta_{-t} \omega\right) \in \mathcal{D}(E) .
$$

Since

$$
\begin{aligned}
\limsup _{t \rightarrow+\infty}( & \left\|\varphi(\tau-t)\left(\theta_{-\tau} \omega\right)\right\|_{E}^{2}+\frac{1}{2}\left(\left\|u_{\tau-t}\right\|_{1}^{2}-p\right)^{2}+k\left\|u_{\tau-t}^{+}\right\|^{2} \\
& \left.+2 C_{3}\left(\left\|u_{\tau-t}\right\|^{2}+\left\|u_{\tau-t}\right\|_{2}^{\gamma+1}+\left\|\phi_{1}\right\|^{2}+\left\|\phi_{3}\right\|_{L_{1}}\right)\right) e^{-\varepsilon_{1} t}=0 .
\end{aligned}
$$

Set

$$
r_{0}^{2}(\omega)=2 a^{2}\left(C_{4} \int_{-\infty}^{0}\left|z\left(\theta_{s} \omega\right)\right|^{\gamma+1} e^{\varepsilon_{1} s} d s+C_{5} \int_{-\infty}^{0}\left|z\left(\theta_{s} \omega\right)\right|^{4} e^{\varepsilon_{1} s} d s+C_{7}\right)<\infty
$$

which is a tempered random variable, then by 4.16) and 4.17), the set $B_{0}(\omega)=\{\varphi \in$ $\left.E:\|\varphi\|_{E} \leq r_{0}(\omega)\right\}$ is a closed measurable absorbing ball in $\mathcal{D}(E)$ and there exists $T(\tau, \omega, B) \geq 0$ such that $\varphi\left(\tau, \tau-t, \theta_{-\tau} \omega, \varphi_{\tau-t}\left(\theta_{-\tau} \omega\right)\right) \in B_{0}(\omega)$ for $t \geq T(\tau, \omega, B)$. The proof is completed.

By (4.1), it concludes that for $\varphi_{\tau-t}\left(\theta_{-\tau} \omega\right) \in B_{0}\left(\theta_{-t} \omega\right)$, and there exist $\left.T\left(\tau, \omega, B_{0}\right)\right) \geq 0$ and a constant $C>0$ such that

$$
\begin{gathered}
\varphi\left(r, \tau-t, \theta_{-\tau} \omega, \varphi_{\tau-t}\left(\theta_{-\tau} \omega\right)\right) \in B_{0}\left(\theta_{r-\tau} \omega\right), \quad \forall t \geq T\left(\tau, \omega, B_{0}\right), \forall r \geq \tau-t \\
\left\|\varphi\left(r, \tau-t, \theta_{-\tau} \omega, \varphi_{\tau-t}\left(\theta_{-\tau} \omega\right)\right)\right\|_{E}^{2} \leq r_{0}^{2}\left(\theta_{r-\tau} \omega\right), \quad \forall r \geq \tau-t, \forall t \geq 0
\end{gathered}
$$

In order to construct the existence of random attracting set for $\Phi$, we have to decompose the solutions of system $(3.3)$ with different initial data into two parts.

For any $\tau \in \mathbb{R}, \omega \in \Omega$ and $t \geq 0$, let $\varphi(r)=\varphi\left(r, \tau-t, \theta_{-\tau} \omega, \varphi_{\tau-t}\left(\theta_{-\tau} \omega\right)\right)(r \geq \tau-t)$ be a mild solution of system (3.3) with the initial value $\varphi_{\tau-t}\left(\theta_{-\tau} \omega\right)=\left(u_{\tau-t}, u_{1, \tau-t}+\right.$ $\left.\varepsilon u_{\tau-t}-a q(x) z\left(\theta_{-t} \omega\right)\right)^{T} \in B_{1}\left(\tau-t, \theta_{-t} \omega\right) \subseteq B_{0}\left(\theta_{-t} \omega\right)$, then by 4.18), it concludes that $\left.\varphi(r) \in B_{0}\left(\theta_{r-\tau} \omega\right)\right)$ for all $r \geq \tau-t$. We decompose $\varphi(r)$ into $\varphi(r)=\varphi_{1}(r)+\varphi_{2}(r)$, where $\varphi_{1}(r)=\left(u_{1}, v_{1}\right)^{T}$ and $\varphi_{2}(r)=\left(u_{2}, v_{2}\right)^{T}$ satisfy

$$
\begin{cases}u_{1 t t}+\triangle^{2} u_{1}+\triangle^{2} u_{1 t}+\left(p-\|\nabla u\|_{L^{2}(U)}^{2}\right) \triangle u_{1}=0 & \text { if } r>\tau-t, \\ \varphi_{1, \tau-t}=\left(u_{1}(\tau-t), v_{1}(\tau-t)\right)^{T}=\left(u_{\tau-t}, u_{1, \tau-t}+\varepsilon u_{\tau-t}\right)^{T} & \text { if } r \leq \tau-t\end{cases}
$$


and

$$
\begin{cases}u_{2 t t}+\triangle^{2} u_{2}+\triangle^{2} u_{2 t}+\left(p-\|\nabla u\|_{L^{2}(U)}^{2}\right) \triangle u_{2}+k u^{+}+f(u, x) & \\ =g(x, t)+a q(x) \dot{W} & \text { if } r>\tau-t \\ \left(u_{2}(\tau-t), v_{2}(\tau-t)\right)^{T}=\left(0,-a q(x) z\left(\theta_{-t} \omega\right)\right)^{T} & \text { if } r \leq \tau-t\end{cases}
$$

respectively.

Next, we will give the exponential decay estimate about $\varphi_{1}$.

Lemma 4.3. Let $p<(1-\varepsilon) \sqrt{\lambda_{1}} / 3$. For any $\tau \in \mathbb{R}, \omega \in \Omega, t \geq 0, r \geq \tau-t$ and $\varphi_{\tau-t}\left(\theta_{-\tau} \omega\right) \in B_{0}\left(\theta_{-t} \omega\right)$, then there exists a random variable $r_{2}(\omega)>0$ such that the solution $\varphi_{1}(r)$ of 4.19 satisfies

$$
\left\|\varphi_{1}\left(r, \tau-t, \varphi_{1, \tau-t}\right)\right\|_{E} \leq r_{1}^{2}(\omega) e^{-\varepsilon(r-\tau+t) / 2}, \quad \forall r \geq \tau-t .
$$

Proof. Taking the inner product in $H$ of 4.19 with $v_{1}\left(r, \tau-t, \varphi_{1, \tau-t}\right)$, in which $v_{1}=$ $u_{1, t}+\varepsilon u_{1}$, we conclude that for $r \geq \tau-t$,

$$
\begin{aligned}
& \frac{1}{2} \frac{d}{d t}\left((1-\varepsilon)\left\|u_{1}\right\|_{2}^{2}+\left\|v_{1}\right\|^{2}\right)+\varepsilon(1-\varepsilon)\left\|u_{1}\right\|_{2}^{2}+\left\|v_{1}\right\|_{2}^{2} \\
&-\varepsilon\left\|v_{1}\right\|^{2}+\varepsilon^{2}\left(u_{1}, v_{1}\right)-\left(p-\|u\|_{1}^{2}\right)\left(A^{1 / 2} u_{1}, v_{1}\right)=0 .
\end{aligned}
$$

Due to 3.1 and 3.2 , we get

$$
\begin{aligned}
& \varepsilon(1-\varepsilon)\left\|u_{1}\right\|_{2}^{2}+\left\|v_{1}\right\|_{2}^{2}-\varepsilon\left\|v_{1}\right\|^{2}+\varepsilon^{2}\left(u_{1}, v_{1}\right) \\
\geq & \varepsilon(1-\varepsilon)\left\|u_{1}\right\|_{2}^{2}+\left\|v_{1}\right\|_{2}^{2}-\varepsilon\left\|v_{1}\right\|^{2}-\frac{\varepsilon(1-\varepsilon)}{2}\left\|u_{1}\right\|_{2}^{2}-\frac{\varepsilon^{3}}{2(1-\varepsilon) \lambda_{1}}\left\|v_{1}\right\|^{2} \\
\geq & \frac{\varepsilon(1-\varepsilon)}{2}\left\|u_{1}\right\|_{2}^{2}+\left(\lambda_{1}-\frac{\varepsilon}{2 \lambda_{1}}-\varepsilon\right)\left\|v_{1}\right\|^{2} \\
\geq & \frac{\varepsilon(1-\varepsilon)}{2}\left\|u_{1}\right\|_{2}^{2}+\frac{\varepsilon}{2}\left\|v_{1}\right\|^{2} .
\end{aligned}
$$

For the last term on the left-hand side of 4.21), we have

$$
\left(\|u\|_{1}^{2}-p\right)\left(A^{1 / 2} u_{1}, v_{1}\right)=\frac{1}{2} \frac{d}{d t}\left(\|u\|_{1}^{2}\left\|u_{1}\right\|_{1}^{2}-p\left\|u_{1}\right\|_{1}^{2}\right)+\varepsilon\left(\|u\|_{1}^{2}\left\|u_{1}\right\|_{1}^{2}-p\left\|u_{1}\right\|_{1}^{2}\right) .
$$

Substituting (4.22) and 4.23) into 4.21) yields, for $r \geq \tau-t$,

$$
\begin{aligned}
& \frac{d}{d t}\left((1-\varepsilon)\left\|u_{1}\right\|_{2}^{2}-p\left\|u_{1}\right\|_{1}^{2}+\left\|v_{1}\right\|^{2}+\|u\|_{1}^{2}\left\|u_{1}\right\|_{1}^{2}\right) \\
+ & \varepsilon\left((1-\varepsilon)\left\|u_{1}\right\|_{2}^{2}-2 p\left\|u_{1}\right\|_{1}^{2}+\left\|v_{1}\right\|^{2}+2\|u\|_{1}^{2}\left\|u_{1}\right\|_{1}^{2}\right) \leq 0 .
\end{aligned}
$$

Let

$$
I(r)=(1-\varepsilon)\left\|u_{1}\right\|_{2}^{2}-p\left\|u_{1}\right\|_{1}^{2}+\left\|v_{1}\right\|^{2}+\|u\|_{1}^{2}\left\|u_{1}\right\|_{1}^{2},
$$


then 3.1 ensures that

$$
\begin{gathered}
I(r) \geq C(p)\left\|u_{1}\right\|_{2}^{2}+\left\|v_{1}\right\|^{2}+\|u\|_{1}^{2}\left\|u_{1}\right\|_{1}^{2} \geq C(p)\left\|\varphi_{1}\right\|_{E}^{2}>0, \\
C(p)= \begin{cases}\frac{1-\varepsilon}{2} & \text { if } p \leq 0, \\
\frac{1-\varepsilon}{2}-\frac{p}{\sqrt{\lambda_{1}}} & \text { if } 0<p \leq(1-\varepsilon) \sqrt{\lambda_{1}} / 3 .\end{cases}
\end{gathered}
$$

Furthermore, we find that

$$
\varepsilon\left((1-\varepsilon)\left\|u_{1}\right\|_{2}^{2}-2 p\left\|u_{1}\right\|_{1}^{2}+\left\|v_{1}\right\|^{2}+2\|u\|_{1}^{2}\left\|u_{1}\right\|_{1}^{2}\right) \geq \frac{\varepsilon}{2} I(r) \quad \text { for } p \leq \frac{(1-\varepsilon) \sqrt{\lambda_{1}}}{3} .
$$

Thus, from 4.24) we conclude that

$$
\frac{d}{d t} I(r)+\frac{\varepsilon}{2} I(r) \leq 0
$$

Noticing that

$$
\left\|\varphi_{1}(r)\right\|_{E}^{2}=\left\|u_{1}\right\|_{2}^{2}+\left\|u_{1, t}+\varepsilon u_{1}\right\|^{2} \leq \frac{I(r)}{C(p)} .
$$

Since $\varphi_{1, \tau-t}=\varphi_{\tau-t}\left(\theta_{-\tau} \omega\right)+\left(0, a q(x) z\left(\theta_{-t} \omega\right)\right)^{T} \in B\left(\theta_{-t} \omega\right)$, by definition of $B_{0}(\omega)$, it follows that

$$
\left\|\varphi_{1, \tau-t}\right\|_{E} \leq r_{0}\left(\theta_{-t} \omega\right)+|a|\|q(x)\| \cdot\left|z\left(\theta_{-t} \omega\right)\right|, \quad \forall \tau \in \mathbb{R}, \omega \in \Omega, t \geq 0 .
$$

Notice that $\varphi_{1, \tau-t}$ is independent of $\omega$, so replacing $\omega$ by $\theta_{t} \omega$ in (4.27), we have

$$
\left\|\varphi_{1, \tau-t}\right\|_{E} \leq r_{0}(\omega)+|a|\|q(x)\| \cdot|z(\omega)|=r_{2}(\omega), \quad \forall \tau \in \mathbb{R}, \omega \in \Omega, t \geq 0 .
$$

By Gronwalll lemma to 4.26) on $[\tau-t, r](r \geq \tau-t)$, we have that for $r \geq \tau-t$,

$$
\begin{aligned}
& \| \varphi_{1}\left(r, \tau-t, \varphi_{1, \tau-t} \|_{E}^{2}\right. \\
\leq & \frac{I(r)}{C(p)} \leq \frac{1}{C(p)} I(\tau-t) e^{-\varepsilon(r-\tau+t) / 2} \\
\leq & \frac{1}{C(p)}\left(\left\|u_{\tau-t}\right\|_{2}^{2}+|p|\left\|u_{\tau-t}\right\|_{1}^{2}+\left\|u_{1, \tau-t}+\varepsilon u_{\tau-t}\right\|^{2}+\left\|u_{\tau-t}\right\|_{1}^{4}\right) e^{-\varepsilon(r-\tau+t) / 2} \\
\leq & \frac{1}{C(p)}\left(\left\|\varphi_{1, \tau-t}\right\|_{E}^{2}+|p|\left\|u_{\tau-t}\right\|_{1}^{2}+\left\|u_{\tau-t}\right\|_{1}^{4}\right) e^{-\varepsilon(r-\tau+t) / 2} \\
\leq & \frac{1}{C(p)}\left(r_{2}^{2}(\omega)+|p| r_{2}^{2}(\omega)+r_{2}^{4}(\omega)\right) e^{-\varepsilon(r-\tau+t) / 2} \leq r_{1}^{2}(\omega) e^{-\varepsilon(r-\tau+t) / 2},
\end{aligned}
$$

we complete the proof.

For the component $\varphi_{2}$ which is ultimately pullback bounded in a "higher regular" space, we have the following estimate. 
Lemma 4.4. Let 1.6) 1.8 hold, $q(x) \in H^{5}(U) \cap H_{0}^{4}(U), p \leq(1-\varepsilon) \sqrt{\lambda_{1}} / 3$. For any $\tau \in \mathbb{R}, \omega \in \Omega$ and $t \geq 0$, there exist a positive constant $\nu \in(0,1 / 4]$ and a random variable $r_{3}(\omega)>0$ such that the solution $\varphi_{2}(r)=\left(u_{2}(r), v_{2}(r)\right)^{T}$ of 4.20 satisfies

$$
\left\|A^{\nu} \varphi_{2}\left(\tau, \tau-t, \theta_{-\tau} \omega, \varphi_{\tau-t}\left(\theta_{-\tau} \omega\right)\right)\right\|_{E}^{2} \leq r_{3}^{2}(\omega)
$$

Proof. We know that $\varphi_{2}=\left(u_{2}, u_{2, t}+\varepsilon u_{2}-a q(x) z\left(\theta_{r-\tau} \omega\right)\right)^{T}$, then equation 4.20) can be rewritten as

$$
\varphi_{2, t}+L \varphi_{2}=F\left(\theta_{r-\tau} \omega, x, r\right), \quad \varphi_{2}\left(r, \tau-t, \theta_{-\tau} \omega, \varphi_{\tau-t}\left(\theta_{-\tau} \omega\right)\right)=\left(0,-a q(x) z\left(\theta_{r-\tau} \omega\right)\right)^{T},
$$

where for $r \geq \tau$,

$$
\begin{aligned}
& F\left(\varphi_{2}, \theta_{r-\tau} \omega, r\right) \\
= & \left(\begin{array}{c}
a q(x) z\left(\theta_{r-\tau} \omega\right) \\
\left(p-\|u\|_{1}^{2}\right) A^{1 / 2} u_{2}-k u^{+}-f(u, x)+g(x, t)-a A q(x) z\left(\theta_{r-\tau} \omega\right)+2 a \varepsilon q(x) z\left(\theta_{r-\tau} \omega\right)
\end{array}\right) .
\end{aligned}
$$

Taking the inner product in $E$ of 4.20 with $A^{2 \nu} \varphi_{2}=\left(A^{2 \nu} u_{2}, A^{2 \nu} v_{2}\right)^{T}$, we obtain

$$
\begin{aligned}
& \quad \frac{1}{2} \frac{d}{d t}\left(\left\|A^{\nu} \varphi_{2}\right\|_{E}^{2}+2 \int_{U} f(u, x) A^{2 \nu} u_{2} d x\right)+\left(L \varphi_{2}, A^{2 \nu} \varphi_{2}\right)_{E} \\
& \quad+\varepsilon \int_{U} f(u, x) A^{2 \nu} u_{2} d x-\int_{U} f_{u}^{\prime}(u, x) u_{t} A^{2 \nu} u_{2} d x \\
& =\left(\left(a q(x) z\left(\theta_{r-\tau} \omega\right), A^{2 \nu} u_{2}\right)\right)+\left(f(u, x), a z\left(\theta_{r-\tau} \omega\right) A^{2 \nu} q(x)\right) \\
& \quad+\left(\left(p-\|u\|_{1}^{2}\right) A^{1 / 2} u_{2}, A^{2 \nu} v_{2}\right)-k\left(u^{+}, A^{2 \nu} v_{2}\right) \\
& \quad+\left(g(x, r)+2 \varepsilon a q(x) z\left(\theta_{r-\tau} \omega\right), A^{2 \nu} v_{2}\right)-\left(a A q(x) z\left(\theta_{r-\tau} \omega\right), A^{2 \nu} v_{2}\right) .
\end{aligned}
$$

Similar to Lemma 4.1, we get

$$
\left(L \varphi_{2}, A^{2 \nu} \varphi_{2}\right)_{E} \geq \frac{\varepsilon}{2}\left\|A^{\nu} \varphi_{2}\right\|_{E}^{2}+\frac{\varepsilon}{4}\left\|A^{\nu} u_{2}\right\|_{2}^{2}+\frac{\lambda_{1}}{2}\left\|A^{\nu} v_{2}\right\|^{2} .
$$

By computation, we have that for $r \geq \tau-t$,

$$
\begin{gathered}
\left(\left(a q(x) z\left(\theta_{r-\tau} \omega\right), A^{2 \nu} u_{2}\right)\right) \leq \frac{\varepsilon}{8}\left\|A^{\nu} u_{2}\right\|_{2}^{2}+\frac{2 a^{2}\left\|A^{\nu} q\right\|_{2}^{2}}{\varepsilon}\left|z\left(\theta_{r-\tau} \omega\right)\right|^{2} \\
\left|-k\left(u^{+}, A^{2 \nu} v_{2}\right)\right| \leq \frac{4 k^{2}}{\lambda_{1}}\left\|A^{\nu} u^{+}\right\|+\frac{\lambda_{1}}{8}\left\|A^{\nu} v_{2}\right\| \\
2 a \varepsilon\left(q(x) z\left(\theta_{r-\tau} \omega\right), A^{2 \nu} v_{2}\right) \leq \frac{8 a^{2} \varepsilon^{2}\left\|A^{\nu} q\right\|^{2}}{\lambda_{1}}\left|z\left(\theta_{r-\tau} \omega\right)\right|^{2}+\frac{\lambda_{1}}{8}\left\|A^{\nu} v_{2}\right\|^{2}, \\
\left|-\left(a A q(x) z\left(\theta_{r-\tau} \omega\right), A^{2 \nu} v_{2}\right)\right| \leq \frac{2 a^{2}\left\|A^{\nu+1} q\right\|^{2}}{\lambda_{1}}\left|z\left(\theta_{r-\tau} \omega\right)\right|^{2}+\frac{\lambda_{1}}{8}\left\|A^{\nu} v_{2}\right\|^{2},
\end{gathered}
$$




$$
\begin{aligned}
& \left(g(x, r), A^{2 \nu} v_{2}\right) \leq \frac{2}{\lambda_{1}}\left\|A^{\nu} g\right\|^{2}+\frac{\lambda_{1}}{8}\left\|A^{\nu} v_{2}\right\|^{2}, \\
& -\left(\left(p-\|u\|_{1}^{2}\right) A^{1 / 2} u_{2}, A^{2 \nu} v_{2}\right) \\
= & -\left(\left(p-\|u\|_{1}^{2}\right) A^{1 / 2} u_{2}, A^{2 \nu}\left(u_{2 t}+\varepsilon u_{2}-q(x) z\left(\theta_{r-\tau} \omega\right)\right)\right) \\
= & \frac{1}{2} \frac{d}{d t}\left(\|u\|_{1}^{2}\left\|u_{2}\right\|_{4 \nu+1}^{2}-p\left\|u_{2}\right\|_{4 \nu+1}^{2}\right)+\varepsilon\left(\|u\|_{1}^{2}\left\|u_{2}\right\|_{4 \nu+1}^{2}-p\left\|u_{2}\right\|_{4 \nu+1}^{2}\right) \\
& +\left(p-\|u\|_{1}^{2}\right)\left(A^{1 / 2} u_{2}, A^{2 \nu} q(x)\right)\left|z\left(\theta_{r-\tau} \omega\right)\right|-\|u\|_{4 \nu+1}^{3}\left\|u_{t}\right\| \\
\geq & \frac{1}{2} \frac{d}{d t}\left(\|u\|_{1}^{2}\left\|u_{2}\right\|_{4 \nu+1}^{2}-p\left\|u_{2}\right\|_{4 \nu+1}^{2}\right)+\varepsilon\left(\|u\|_{1}^{2}\left\|u_{2}\right\|_{4 \nu+1}^{2}-p\left\|u_{2}\right\|_{4 \nu+1}^{2}\right) \\
& -\frac{\varepsilon}{2}|p|\left\|u_{2}\right\|_{4 \nu+1}^{2}-\left(|p|+\|u\|_{1}^{2}\right)\left\|u_{2}\right\|\left\|_{4 \nu+1}\right\| A^{1 / 2} q(x)\left\|\left|z\left(\theta_{r-\tau} \omega\right)\right|-\right\| u\left\|_{4 \nu+1}^{3}\right\| u_{t} \| \\
\geq & \frac{1}{2} \frac{d}{d t}\left(\|u\|_{1}^{2}\left\|u_{2}\right\|_{4 \nu+1}^{2}-p\left\|u_{2}\right\|_{4 \nu+1}^{2}\right)+\varepsilon\left(\|u\|_{1}^{2}\left\|u_{2}\right\|_{4 \nu+1}^{2}-p\left\|u_{2}\right\|_{4 \nu+1}^{2}\right) \\
& -\left(\frac{\varepsilon}{2}|p|+\frac{1}{2}\left(|p|+\|u\|_{1}^{2}\right)^{2}\right)\left\|u_{2}\right\|_{4 \nu+1}^{2}-\frac{1}{2}\left\|A^{1 / 2} q\right\|^{2}\left|z\left(\theta_{r-\tau} \omega\right)\right|^{2}-\|u\|_{4 \nu+1}^{3}\left\|u_{t}\right\| .
\end{aligned}
$$

From (1.6) and 4.18), we have that for $r \geq \tau-t$,

$$
\begin{aligned}
& \left(f(u, x), a z\left(\theta_{r-\tau} \omega\right) A^{2 \nu} q(x)\right) \\
\leq & \left.\int_{U}\left(C_{0}|u|^{\gamma}+\left|\phi_{1}(x)\right|\right) \mid a z\left(\theta_{r-\tau} \omega\right) A^{2 \nu} q(x)\right) \mid d x \\
\leq & \left.\left.C_{0}|a|\|u\|^{\gamma} \| z\left(\theta_{r-\tau} \omega\right) A^{2 \nu} q(x)\right)\|+|a|\| \phi_{1}(x)\|\| z\left(\theta_{r-\tau} \omega\right) A^{2 \nu} q(x)\right) \| \\
\leq & \left.\frac{C_{0}^{2} a^{2}}{2}\|u\|^{2 \gamma}+\frac{1}{2}\left\|\phi_{1}(x)\right\|^{2}+|a| \| z\left(\theta_{r-\tau} \omega\right) A^{2 \nu} q(x)\right) \|^{2} \\
\leq & \frac{C_{0}^{2} a^{2}}{2} r_{0}^{2 \gamma}\left(\theta_{r-\tau} \omega\right)+\frac{1}{2}\left\|\phi_{1}(x)\right\|^{2}+a^{2}\|q\|_{4 \nu+1}^{2}\left|z\left(\theta_{r-\tau} \omega\right)\right|^{2},
\end{aligned}
$$

by (1.6), 4.18 and the Sobolev embedding theorem, we know that $f_{u}^{\prime}(u, x)$ is uniformly bounded in $L^{\infty}$. That is,

$$
\left|f_{u}^{\prime}(u, x)\right|_{L^{\infty}} \leq C_{0}|u|_{L^{\infty}}^{\gamma-1}+\left|\phi_{1}(x)\right|_{L^{\infty}} \leq C_{8}\|u\|_{2}^{\gamma-1}+\left\|\phi_{1}(x)\right\| \leq C_{9} r_{0}^{\gamma-1}\left(\theta_{r-\tau} \omega\right) .
$$

Combining with the Hölder, Cauchy inequalities and (4.18), we conclude that

$$
\begin{aligned}
\int_{U} f_{u}^{\prime}(u, x) u_{t} A^{2 \nu} u_{2} d x & \leq C_{9} r_{0}^{\gamma-1}\left(\theta_{r-\tau} \omega\right) \int_{U}\left|u_{t}\right| \cdot\left|A^{2 \nu} u_{2}\right| d x \\
& \leq C_{9} r_{0}^{\gamma-1}\left(\theta_{r-\tau} \omega\right)\left(\int_{U}\left|u_{t}\right|^{2} d x\right)^{1 / 2}\left(\int_{U}\left|A^{2 \nu} u_{2}\right|^{2} d x\right)^{1 / 2} \\
& \leq C_{10} r_{0}^{\gamma-1}\left(\theta_{r-\tau} \omega\right)\left\|u_{t}\right\| \cdot\left\|A^{\nu} u_{2}\right\|_{2} \\
& \leq C_{11} r_{0}^{2 \gamma}\left(\theta_{r-\tau} \omega\right)+\frac{\varepsilon}{8}\left\|A^{\nu} u_{2}\right\|_{2}^{2}
\end{aligned}
$$


Therefore, collecting all 4.30-4.39), from 4.29 yields, for $r \geq \tau-t$,

$$
\begin{aligned}
& \quad \frac{d}{d t}\left(\left\|A^{\nu} \varphi_{2}\right\|_{E}^{2}+\|u\|_{1}^{2}\left\|u_{2}\right\|_{4 \nu+1}^{2}-p\left\|u_{2}\right\|_{4 \nu+1}^{2}+2 \int_{U} f(u, x) A^{2 \nu} u_{2} d x\right) \\
& +\varepsilon\left(\left\|A^{\nu} \varphi_{2}\right\|_{E}^{2}+\|u\|_{1}^{2}\left\|u_{2}\right\|_{4 \nu+1}^{2}-p\left\|u_{2}\right\|_{4 \nu+1}^{2}+2 \int_{U} f(u, x) A^{2 \nu} u_{2} d x\right) \\
& \leq C_{11}\left(a^{2}\left|z\left(\theta_{r-\tau} \omega\right)\right|^{2}+a^{2} r_{0}^{2 \gamma}\left(\theta_{r-\tau} \omega\right)+r_{0}^{6}\left(\theta_{r-\tau} \omega\right)+\left\|\phi_{1}(x)\right\|\right) .
\end{aligned}
$$

Let

$$
\begin{gathered}
\chi_{1}(r)=\left\|A^{\nu} \varphi_{2}\right\|_{E}^{2}+\|u\|_{1}^{2}\left\|u_{2}\right\|_{4 \nu+1}^{2}-p\left\|u_{2}\right\|_{4 \nu+1}^{2}+2 \int_{U} f(u, x) A^{2 \nu} u_{2} d x, \\
m_{2}\left(\theta_{r-\tau} \omega\right)=C_{12}\left(a^{2}\left|z\left(\theta_{r-\tau} \omega\right)\right|^{2}+a^{2} r_{0}^{2 \gamma}\left(\theta_{r-\tau} \omega\right)+r_{0}^{6}\left(\theta_{r-\tau} \omega\right)+\left\|\phi_{1}(x)\right\|\right) .
\end{gathered}
$$

It follows from 4.40, 4.41 and 4.42 that

$$
\frac{d}{d t} \chi_{1}(r)+\varepsilon \chi_{1}(r) \leq m_{2}\left(\theta_{r-\tau} \omega\right), \quad \forall r \geq \tau-t .
$$

Note that $\chi_{1}\left(\tau-t, \tau-t, \theta_{-\tau} \omega, \varphi_{\tau-t}\left(\theta_{-\tau} \omega\right)\right) \leq a^{2}\left\|A^{\nu} q\right\|^{2}\left|z\left(\theta_{r-\tau} \omega\right)\right|^{2}$, then by applying Gronwall lemma to 4.43$)$ on $[\tau-t, r](r \geq \tau-t)$, we have

$$
\chi_{1}\left(r, \tau-t, \theta_{-\tau} \omega, \varphi_{\tau-t}\left(\theta_{-\tau} \omega\right)\right) \leq \int_{\tau-t}^{r} m_{2}\left(\theta_{s-\tau} \omega\right) e^{-\varepsilon(r-s)} d s, \quad \forall r \geq \tau-t .
$$

From (1.6) and 4.18), we have that for $r \geq \tau-t$,

$$
\begin{aligned}
\left|\int_{U} f(u, x) A^{2 \nu} u_{2} d x\right| & \leq \int_{U}\left(C_{0}|u|^{\gamma}+\left|\phi_{1}(x)\right|\right)\left|A^{2 \nu} u_{2}\right| d x \\
& \leq C_{0}\|u\|^{\gamma}\left\|A^{2 \nu} u_{2}\right\|+\left\|\phi_{1}(x)\right\|\left\|A^{2 \nu} u_{2}\right\| \\
& \leq C_{13}\left(r_{0}^{2 \gamma}\left(\theta_{r-\tau} \omega\right)+\left\|\phi_{1}(x)\right\|^{2}\right)+\frac{1-\varepsilon}{4}\left\|A^{\nu} u_{2}\right\|_{2}^{2} .
\end{aligned}
$$

It then follows from 4.41 and 4.45 that

$$
\chi_{1}(r) \geq C(p)\left\|A^{\nu} \varphi_{2}\right\|_{E}^{2}-C_{13}\left(r_{0}^{2 \gamma}\left(\theta_{r-\tau} \omega\right)+\left\|\phi_{1}(x)\right\|^{2}\right),
$$

where $C(p)$ is defined as in 4.25). Therefore, by 4.44 and 4.46), we find that for $t \geq 0$,

$$
\begin{aligned}
& \left\|A^{\nu} \varphi_{2}\left(\tau, \tau-t, \theta_{-\tau} \omega, \varphi_{\tau-t}\left(\theta_{-\tau} \omega\right)\right)\right\|_{E}^{2} \\
\leq & \frac{1}{C(p)} \chi_{1}\left(\tau, \tau-t, \theta_{-\tau} \omega, \varphi_{\tau-t}\left(\theta_{-\tau} \omega\right)\right)+\frac{C_{12}}{C(p)}\left(r_{0}^{2 \gamma}(\omega)+\left\|\phi_{1}(x)\right\|^{2}\right) \\
\leq & \frac{a^{2}\left\|A^{\nu} q\right\|^{2}\left|z\left(\theta_{r-\tau} \omega\right)\right|^{2}}{C(p)} e^{-\varepsilon t} \\
& +\frac{C_{14}}{C(p)} \int_{\tau-t}^{\tau}\left(a^{2}\left|z\left(\theta_{s-\tau} \omega\right)\right|^{2}+a^{2} r_{0}^{2 \gamma}\left(\theta_{s-\tau} \omega\right)+r_{0}^{6}\left(\theta_{s-\tau} \omega\right)+\left\|\phi_{1}(x)\right\|\right) e^{-\varepsilon(\tau-s)} d s \\
& +\frac{C_{13}}{C(p)}\left(r_{0}^{2 \gamma}(\omega)+\left\|\phi_{1}(x)\right\|^{2}\right) .
\end{aligned}
$$


Taking

$$
\begin{aligned}
r_{3}^{2}(\omega)= & \frac{2 C_{14}}{C(p)} \int_{-\infty}^{0}\left(a^{2}\left|z\left(\theta_{s} \omega\right)\right|^{2}+a^{2} r_{0}^{2 \gamma}\left(\theta_{s} \omega\right)+r_{0}^{6}\left(\theta_{s} \omega\right)+\left\|\phi_{1}(x)\right\|\right) e^{\varepsilon s} d s \\
& +\frac{2 C_{13}}{C(p)}\left(r_{0}^{2 \gamma}(\omega)+\left\|\phi_{1}(x)\right\|^{2}\right),
\end{aligned}
$$

then 4.47) and 4.48 imply 4.28). The proof is completed.

We have our main result about the existence of a random attractor for the RDS $\Phi$ as follows.

Theorem 4.5. Assume the conditions 1.6 -1.8 hold, $q(x) \in H^{5}(U) \cap H_{0}^{4}(U), p \leq$ $(1-\varepsilon) \sqrt{\lambda_{1}} / 3$, then for any $\tau \in \mathbb{R}, \omega \in \Omega$, the RDS $\Phi$ associated with (3.3) possesses a compact measurable $\mathcal{D}(E)$-pullback attracting set $\Upsilon(\tau, \omega) \subset E$ and a $\mathcal{D}(E)$-pullback random attractor $\mathcal{A}(\tau, \omega) \subseteq \cap B_{0}(\omega)$.

Proof. For any $\tau \in \mathbb{R}, \omega \in \Omega$, let $\Upsilon(\tau, \omega)$ be the closed ball of $\mathcal{H}^{8 \nu+1} \times \mathcal{H}^{8 \nu}$ of radius $r_{3}(\omega)$, where $\nu \in(0,1 / 4]$, then $\Upsilon(\tau, \omega) \in \mathcal{D}(E)$. Since the embedding $\mathcal{H}^{8 \nu+1} \times \mathcal{H}^{8 \nu} \hookrightarrow\left(H^{2}(U) \cap\right.$ $\left.H_{0}^{1}(U)\right) \times L^{2}(U)$ is compact, that is, $\Upsilon(\tau, \omega)$ is compact in $\left(H^{2}(U) \cap H_{0}^{1}(U)\right) \times L^{2}(U)$. Therefore, $\Upsilon(\tau, \omega)$ is compact in $E$.

For every $\Upsilon(\tau, \omega) \in \mathcal{D}(E)$, we know that

$$
\lim _{t \rightarrow \infty} d\left(\Phi\left(t, \tau-t, \theta_{-t} \omega, B\left(\tau-t, \theta_{-t} \omega\right)\right), \Upsilon(\tau, \omega)\right)=0
$$

By Lemma 4.2, there exists $t^{*}=t^{*}(\tau, \omega, B) \geq 0$ such that

$$
\varphi\left(\tau, \tau-t, \theta_{-\tau} \omega, B\left(\tau-t, \theta_{-\tau} \omega\right)\right) \subseteq B_{0}(\omega), \quad \forall t>t^{*}
$$

Let $t>t^{*}$ and $t_{0}=t-t^{*}>T\left(\tau, \omega, B_{0}\right) \geq 0$. Using the cocycle property (iii) of $\Phi$, we conclude that

$$
\begin{aligned}
& \varphi\left(\tau, \tau-t, \theta_{-\tau} \omega, B\left(\tau-t, \theta_{-\tau} \omega\right)\right) \\
= & \varphi\left(\tau, \tau-t_{0}-t^{*}, \theta_{-\tau} \omega, B\left(\tau-t_{0}-t^{*}, \theta_{-\tau} \omega\right)\right) \\
= & \varphi\left(\tau, \tau-t_{0}, \theta_{-\tau} \omega, \varphi\left(\tau-t_{0}, \tau-t_{0}-t^{*}, \theta_{-\tau} \omega\right), B\left(\tau-t_{0}-t^{*}, \theta_{-\tau} \omega\right)\right) \\
\subseteq & \varphi\left(\tau, \tau-t_{0}, \theta_{-\tau} \omega, B_{0}\left(\theta_{-t_{0}} \omega\right)\right) \subseteq B_{1}(\tau, \omega) .
\end{aligned}
$$

Taking any $\varphi\left(\tau, \tau-t, \theta_{-\tau} \omega, \varphi_{\tau-t}\left(\theta_{-\tau} \omega\right)\right) \in \varphi\left(\tau, \tau-t, \theta_{-\tau} \omega, B\left(\tau-t, \theta_{-t} \omega\right)\right)$ for $t>t^{*}+$ $T\left(\tau, \omega, B_{0}\right)$, where $\varphi_{\tau-t}\left(\theta_{-\tau} \omega\right) \in B\left(\tau-t, \theta_{-t} \omega\right)$. By 4.49) and Lemma 4.4. we have

$$
\begin{aligned}
& \varphi_{2}\left(\tau, \tau-t, \theta_{-\tau} \omega, \varphi_{\tau-t}\left(\theta_{-\tau} \omega\right)\right) \\
= & \varphi\left(\tau, \tau-t, \theta_{-\tau} \omega, \varphi_{\tau-t}\left(\theta_{-\tau} \omega\right)\right)-\varphi_{1}\left(\tau, \tau-t, \theta_{-\tau} \omega, \varphi_{1, \tau-t}\left(\theta_{-\tau} \omega\right)\right) \\
\in & \Upsilon(\tau, \omega) .
\end{aligned}
$$


Thus, by Lemma 4.3 , we find

$$
\begin{aligned}
\inf _{\xi \in B(\tau, \omega)}\left\|\varphi\left(\tau, \tau-t, \theta_{-\tau} \omega, \varphi_{\tau-t}\left(\theta_{-\tau} \omega\right)\right)-\xi\right\|_{E}^{2} & \leq \varphi_{1}\left(\tau, \tau-t, \theta_{-\tau} \omega, \varphi_{1, \tau-t}\left(\theta_{-\tau} \omega\right)\right) \\
& \leq r_{1}^{2}(\omega) e^{-\varepsilon t / 2}, \quad \forall t>t^{*}+T\left(\tau, \omega, B_{0}\right) .
\end{aligned}
$$

It follows that

$$
\lim _{t \rightarrow \infty} d\left(\varphi\left(\tau, \tau-t, \theta_{-\tau} \omega, B\left(\tau-t, \theta_{-\tau} \omega\right)\right), \Upsilon(\tau, \omega)\right) \leq r_{1}^{2}(\omega) e^{-\varepsilon t / 2} \rightarrow 0 \quad \text { as } t \rightarrow+\infty .
$$

From Theorem 2.8 and Lemma 4.2 , the $\operatorname{RDS} \Phi$ associated with $(3.3)$ possesses a $\mathcal{D}(E)$ pullback random attractor $\mathcal{A}(\tau, \omega) \subseteq B(\tau, \omega) \cap B_{0}(\omega)$. The proof is completed.

\section{Upper semicontinuity of random attractors}

We can define a family of random dynamical systems $\left\{\Phi^{a}(t, \tau, \omega)\right\}_{a \in \mathbb{R}}$ associated to 3.3. by Theorem 4.5, we know that $\left\{\Phi^{a}(t, \tau, \omega)\right\}_{a \in \mathbb{R}}$ possess a corresponding family of random attractors $\left\{\mathcal{A}^{a}(\tau, \omega)\right\}_{a \in \mathbb{R}}$. In this section, we consider the upper semicontinuity of random attractors $\left\{\mathcal{A}^{a}(\tau, \omega)\right\}_{a \in \mathbb{R}}$ as $a \rightarrow 0$ by Theorem 2.9 .

When $a=0$, the system 3.3 reduces to a deterministic non-autonomous one in $E$ :

$$
\dot{\widetilde{\varphi}}+L \widetilde{\varphi}=\widetilde{F}(\widetilde{\varphi}, t), \quad \widetilde{\varphi}_{\tau}=\left(\widetilde{u}_{\tau}, \widetilde{u}_{1, \tau}+\varepsilon \widetilde{u}_{\tau}\right)^{T}, \quad t \geq \tau, \tau \in \mathbb{R}
$$

where

$$
\begin{aligned}
\widetilde{\varphi}(t, \tau, x) & =\left(\begin{array}{l}
\widetilde{u} \\
\widetilde{v}
\end{array}\right)=\left(\begin{array}{c}
\widetilde{u} \\
\widetilde{u}_{t}+\varepsilon \widetilde{u}
\end{array}\right), \\
\widetilde{F}(\widetilde{\varphi}, t) & =\left(\begin{array}{c}
0 \\
-\left(p-\|\widetilde{u}\|_{1}^{2}\right) A^{1 / 2} \widetilde{u}-k \widetilde{u}^{+}-f(\widetilde{u}, x)+g(x, t) \\
0
\end{array}\right) .
\end{aligned}
$$

Notice that under condition (1.6) 1.8 , the solutions $\widetilde{\varphi}\left(t, \tau, x, \widetilde{\varphi}_{0}\right)$ of $(5.1)$ generates a continuous process $\widetilde{\Phi}(t, \tau): \widetilde{\varphi}_{\tau} \rightarrow \widetilde{\varphi}\left(t, \tau, x, \widetilde{\varphi}_{0}\right), E \rightarrow E, t \geq \tau$. Similar to Sections 3 , for any $\tau \in \mathbb{R}$ and for every $B \subset E$, we can prove that $\{\widetilde{\Phi}(t, \tau)\}_{t \geq \tau}$ has a uniform bounded absorbing set $\widetilde{B}=\left\{\widetilde{\varphi} \in E:\|\widetilde{\varphi}\|_{E} \leq \widetilde{r}_{0}\right\}$, where $\widetilde{r}_{0}>0$ is independent of $\tau$. There exists $T(B, \tau)>0$ such that $\widetilde{\Phi}(t, \tau) B \subseteq \widetilde{B}$ for all $t \geq T(B, \tau)+\tau$, the process $\{\widetilde{\Phi}(t, \tau)\}_{t \geq \tau}$ has a pullback attractor $\{\widetilde{\mathcal{A}}(\tau)\}_{\tau \in \mathbb{R}}$ with properties as follows:

(a) $\{\widetilde{\mathcal{A}}(\tau)\}_{\tau \in \mathbb{R}}$ is compact and $\{\widetilde{\mathcal{A}}(\tau)\}_{\tau \in \mathbb{R}} \subseteq \widetilde{B}$ for each $\tau \in \mathbb{R}$;

(b) $\widetilde{\Phi}(t, \tau) \widetilde{\mathcal{A}}(\tau)=\widetilde{\mathcal{A}}(t)$ for $t \geq \tau$; 
(c) for any $\tau \in \mathbb{R}$ and any bounded set $B \subset E, \lim _{t \rightarrow+\infty} d(\widetilde{\Phi}(\tau, \tau-t) B, \widetilde{\mathcal{A}}(\tau))=0$.

Theorem 5.1. Assume the conditions $1.6-1.8$ hold, then for any $\tau \in \mathbb{R}, \omega \in \Omega$,

$$
\lim _{a \rightarrow 0} d\left(\mathcal{A}^{a}(\tau, \omega), \widetilde{\mathcal{A}}(\tau)\right)=\sup _{\varphi \in \mathcal{A}^{a}(\tau, \omega)} \inf _{\widetilde{\varphi} \in \widetilde{\mathcal{A}}(\tau)}\|\varphi-\widetilde{\varphi}\|_{E}=0
$$

Proof. We check that $\left\{\mathcal{A}^{a}(\tau, \omega)\right\}_{a \in \mathbb{R}}$ and $\{\widetilde{\mathcal{A}}(\tau)\}_{\tau \in \mathbb{R}}$ satisfy conditions (i)-(iv) of Theorem 2.9 one by one.

(i) It holds from above statements obviously.

(ii) From Lemma 4.2 and Theorem 4.5, for any $\tau \in \mathbb{R}, \omega \in \Omega$ and $a \in \mathbb{R}$, the absorbing ball $B_{0}(a, \omega)=\left\{\varphi \in E:\|\varphi\|_{E} \leq r_{0}(a, \omega)\right\}$ includes the random attractor $\mathcal{A}^{a}(\tau, \omega)$ for $\Phi^{a}(t, \tau, \omega)$, that is, $\mathcal{A}^{a}(\tau, \omega) \subseteq B_{0}(a, \omega) \subset E$, where $r_{0}(a, \omega)$ is defined by (4.17) as follows:

$$
\begin{aligned}
r_{0}^{2}(a, \omega) & =2\left(C_{4}|a| \int_{-\infty}^{0}\left|z\left(\theta_{s} \omega\right)\right|^{\gamma+1} e^{\varepsilon_{1} s} d s+C_{6}+2\left\|\phi_{2}\right\|_{L^{1}}\right) \\
& <\widetilde{r}_{0}^{2}+2 C_{4}|a| \int_{-\infty}^{0}\left|z\left(\theta_{s} \omega\right)\right|^{\gamma+1} e^{\varepsilon_{1} s} d s
\end{aligned}
$$

that is,

$$
\limsup _{a \rightarrow 0} r_{0}^{2}(a, \omega) \leq \widetilde{r}_{0}^{2}
$$

(iii) Let $|a| \leq 1$. For every $\tau \in \mathbb{R}, \omega \in \Omega$, by Theorem 4.5 , we have that $\mathcal{A}^{a}(\tau, \omega) \subseteq$ $\Upsilon^{a}(\tau, \omega) \subset E$. In fact, note that $r_{3}^{2}(a, \omega)$ and $r_{0}^{2}(a, \omega)$ are both increasing functions in $|a|$. By the construction of $\Upsilon^{a}(\tau, \omega)$ in Theorem 4.5, we can choose the compact set $\Upsilon^{a}(\tau, \omega)$ satisfying

$$
\Upsilon^{a}(\tau, \omega) \subseteq \Upsilon^{1}(\tau, \omega), \quad \forall|a| \leq 1
$$

Thus,

$$
\bigcup_{|a| \leq 1} \mathcal{A}^{a}(\tau, \omega) \subseteq \bigcup_{|a| \leq 1} \Upsilon^{a}(\tau, \omega) \subseteq \Upsilon^{1}(\tau, \omega) \subset E .
$$

Therefore $\bigcup_{|a| \leq 1} \mathcal{A}^{a}(\tau, \omega)$ is precompact in $E$.

(iv) Let $|a| \leq 1$. For every $\tau \in \mathbb{R}, \omega \in \Omega, t \geq 0$, and $r \geq \tau-t$, suppose that $\varphi^{a}\left(r, \tau-t, \theta_{-\tau} \omega, \varphi_{\tau-t}^{a}\left(\theta_{-\tau} \omega\right)\right)$ and $\widetilde{\varphi}\left(r, \tau-t, \widetilde{\varphi}_{\tau-t}\right)$ are the solutions of (3.3) and (5.1) with initial data $\varphi_{\tau-t}^{a}\left(\theta_{-\tau} \omega\right)$ and $\widetilde{\varphi}_{\tau-t}$, respectively. Let $\widehat{\varphi}=\varphi^{a}-\widetilde{\varphi}=(\widehat{u}, \widehat{v})=\left(u^{a}-\widetilde{u}, v^{a}-\widetilde{v}\right)$, then

$$
\begin{aligned}
\dot{\hat{\varphi}}+L \widehat{\varphi} & =F^{a}\left(\varphi^{a}, \theta_{r-\tau} \omega, x, r\right)-\widetilde{F}(\widetilde{\varphi}, x, r), \\
\widehat{\varphi}\left(\theta_{-\tau} \omega\right) & =\varphi_{\tau-t}^{a}\left(\theta_{-\tau} \omega\right)-\widetilde{\varphi}_{\tau-t}, \quad \forall \geq \tau-t,
\end{aligned}
$$


where

$$
\begin{gathered}
F^{a}\left(\varphi^{a}, \theta_{r-\tau} \omega, x, r\right)-\widetilde{F}(\widetilde{\varphi}, x, r) \\
=\left(\begin{array}{c}
a q(x) z\left(\theta_{r-\tau} \omega\right) \\
\left(p-\left\|u^{a}\right\|_{1}^{2}\right) A^{1 / 2} u^{a}-\left(p-\|\widetilde{u}\|_{1}^{2}\right) A^{1 / 2} \widetilde{u}-k \widehat{u}^{+}+f(\widetilde{u}, x) \\
-f\left(u^{a}, x\right)-a A q(x) z\left(\theta_{r-\tau} \omega\right)+2 a \varepsilon q(x) z\left(\theta_{r-\tau} \omega\right)
\end{array}\right), \\
\widehat{\varphi}\left(\theta_{-\tau} \omega\right)=\left(\begin{array}{c}
u_{\tau}^{a}-\widetilde{u}_{\tau} \\
u_{1, \tau}^{a}-\widetilde{u}_{1, \tau}+\varepsilon\left(u_{\tau}^{a}-\widetilde{u}_{\tau}\right)-a q(x) z\left(\theta_{\tau} \omega\right)
\end{array}\right) .
\end{gathered}
$$

Taking the inner product of system $\sqrt{5.2}$ with $\widehat{\varphi}$ in $E$, we find

$$
\begin{aligned}
& \frac{1}{2} \frac{d}{d t}\|\widehat{\varphi}\|_{E}^{2}+\frac{\varepsilon}{2}\|\widehat{\varphi}\|_{E}^{2}+\frac{\varepsilon}{4}\|\widehat{u}\|_{2}^{2}+\frac{\lambda_{1}}{2}\|\widehat{v}\|^{2} \\
\leq & \left(\left(a q(x) z\left(\theta_{r-\tau} \omega\right), \widehat{u}\right)\right)+\left(\left(p-\left\|u^{a}\right\|_{1}^{2}\right) A^{1 / 2} u^{a}-\left(p-\|\widetilde{u}\|_{1}^{2}\right) A^{1 / 2} \widetilde{u}, \widehat{v}\right)-k\left(\widehat{u}^{+}, \widehat{v}\right) \\
& +\left(f(\widetilde{u}, x)-f\left(u^{a}, x\right), \widehat{v}\right)+\left(a A q(x) z\left(\theta_{r-\tau} \omega\right), \widehat{v}\right)+\left(2 a \varepsilon q(x) z\left(\theta_{r-\tau} \omega\right), \widehat{v}\right) .
\end{aligned}
$$

We deal with the terms in the right side of $(5.3)$ one by one as follows:

$$
\begin{gathered}
\left(\left(a q(x) z\left(\theta_{r-\tau} \omega\right), u\right)\right) \leq \frac{1}{2}\left(\|\widehat{u}\|_{2}^{2}+a^{2}\|q\|_{2}^{2}\left|z\left(\theta_{r-\tau} \omega\right)\right|^{2}\right), \\
\left|-k\left(\widehat{u}^{+}, \widehat{v}\right)\right| \leq \frac{k^{2}}{2}\|\widehat{u}\|_{2}^{2}+\frac{1}{2}\|\widehat{v}\|^{2}, \\
\left.\mid a A q(x) z\left(\theta_{r-\tau} \omega\right), \widehat{v}\right)\left.\left|\leq \frac{2 a^{2}\|q\|_{4}^{2}}{\lambda_{1}}\right| z\left(\theta_{r-\tau} \omega\right)\right|^{2}+\frac{\lambda_{1}}{8}\|\widehat{v}\|^{2}, \\
\left(2 a \varepsilon q(x) z\left(\theta_{r-\tau} \omega\right), \widehat{v}\right) \leq \frac{8 a^{2} \varepsilon^{2}\|q\|^{2}}{\lambda_{1}}\left|z\left(\theta_{r-\tau} \omega\right)\right|^{2}+\frac{\lambda_{1}}{8}\|\widehat{v}\|^{2}, \\
\left|\left(\left(p-\left\|u^{a}\right\|_{1}^{2}\right) A^{1 / 2} u^{a}-\left(p-\|\widetilde{u}\|_{1}^{2}\right) A^{1 / 2} \widetilde{u}, \widehat{v}\right)\right| \\
\leq \frac{4}{\lambda_{1}}\left[\left(p-\left\|u^{a}\right\|_{1}^{2}\right)^{2}\left\|u^{a}\right\|_{2}^{2}+\left(p-\|\widetilde{u}\|_{1}^{2}\right)^{2}\|\widetilde{u}\|_{2}^{2}\right]+\frac{\lambda_{1}}{8}\|\widehat{v}\|^{2} \\
\leq C_{14} r_{0}^{6}\left(\theta_{r-\tau} \omega\right)+C_{16}+\frac{\lambda_{1}}{8}\|\widehat{v}\|^{2} \leq a^{12} C_{167} r_{4}^{6}\left(\theta_{r-\tau} \omega\right)+\frac{\lambda_{1}}{8}\|\widehat{v}\|^{2},
\end{gathered}
$$

where $C_{17}$ and $r_{4}\left(\theta_{r-\tau} \omega\right)$ are independent of $a$. By $(1.6)$, Hölder inequality and the Sobolev embedding theorem, we obtain

$$
\begin{aligned}
& -\left(f(\widetilde{u}, x)-f\left(u^{a}, x\right), x, \widehat{v}\right) \\
\leq & \frac{2}{\lambda_{1}}\left\|\left(f(\widetilde{u}, x)-f\left(u^{a}, x\right), x\right)\right\|^{2}+\frac{\lambda_{1}}{8}\|\widehat{v}\|^{2} \\
\leq & \frac{2}{\lambda_{1}} C_{0} \int_{U}\left(\left|\phi_{1}(x)\right|+|\widetilde{u}|^{2(\gamma-1)}+\left|u^{a}\right|^{2(\gamma-1)}\right)|\widehat{u}|^{2} d x+\frac{\lambda_{1}}{8}\|\widehat{v}\|^{2} \\
\leq & \frac{2}{\lambda_{1}} C_{17}\left(\int_{U}\left(\left|\phi_{1}(x)\right|+|\widetilde{u}|^{2(\gamma-1)}+\left|u^{a}\right|^{2(\gamma-1)}\right)^{3 / 2} d x\right)^{2 / 3}\left(\int_{U}|\widehat{u}|^{6} d x\right)^{1 / 3}
\end{aligned}
$$




$$
\begin{aligned}
& +\frac{\lambda_{1}}{8}\|\widehat{v}\|^{2} \\
\leq & C_{19}\left(\left\|\phi_{1}(x)\right\|^{2}+\|\widetilde{u}\|_{2}^{2(\gamma-1)}+\left\|u^{a}\right\|_{2}^{2(\gamma-1)}\right)\|\widehat{u}\|_{2}^{2}+\frac{\lambda_{1}}{8}\|\widehat{v}\|^{2} \\
\leq & C_{20} r_{5}^{2(\gamma-1)}\left(\theta_{r-\tau} \omega\right)\|\widehat{u}\|_{2}^{2}+\frac{\lambda_{1}}{8}\|\widehat{v}\|^{2},
\end{aligned}
$$

where $C_{20}$ and $r_{5}\left(\theta_{r-\tau} \omega\right)$ are independent of $a$. Together with $(5.3)-(5.9)$, it follows that

$$
\frac{d}{d t}\|\widehat{\varphi}\|_{E}^{2}=\left(C_{20} r_{5}^{2(\gamma-1)}\left(\theta_{r-\tau} \omega\right)+C_{21}\right)\|\widehat{\varphi}\|_{E}^{2}+a^{2} \cdot C_{22}\left|z\left(\theta_{r-\tau} \omega\right)\right|^{2}, \quad r \geq \tau-t
$$

where $C_{21}, C_{22}$ are independent of $a$. Notice that

$$
\begin{aligned}
\|\widehat{\varphi}(r, \tau-t)\|_{E}^{2} & =\left\|\varphi^{a}\left(r, \tau-t, \theta_{-\tau} \omega, \varphi_{\tau-t}^{a}\left(\theta_{-\tau} \omega\right)\right)-\widetilde{\varphi}\left(r, \tau-t, \widetilde{\varphi}_{\tau-t}\right)\right\|_{E}^{2} \\
& =\|\widehat{u}\|_{2}^{2}+\|\widehat{v}\|^{2}, \quad r \geq \tau-t .
\end{aligned}
$$

By Gronwall lemma, we find

$$
\begin{gathered}
\left\|\varphi^{a}\left(\tau, \tau-t, \theta_{-\tau} \omega, \varphi_{\tau-t}^{a}\left(\theta_{-\tau} \omega\right)\right)-\widetilde{\varphi}\left(\tau, \tau-t, \widetilde{\varphi}_{\tau-t}\right)\right\|_{E}^{2} \\
\leq\left\|\varphi_{\tau-t}^{a}\left(\theta_{-\tau} \omega\right)-\widetilde{\varphi}_{\tau-t}\right\|_{E}^{2} e^{\int_{\tau-t}^{\tau}\left(C_{20} r_{5}^{2(\gamma-1)}\left(\theta_{r-\tau} \omega\right)+C_{21}\right)} d r \\
\quad+a^{2} C_{22} \int_{\tau-t}^{\tau}\left|z\left(\theta_{r-\tau} \omega\right)\right|^{2} e^{\int_{r}^{\tau}\left(C_{20} r_{5}^{2(\gamma-1)}\left(\theta_{\sigma-\tau} \omega\right)+C_{21}\right)} d \sigma d r .
\end{gathered}
$$

For any $\tau \in \mathbb{R}, \omega \in \Omega, t \geq 0, a_{n} \rightarrow 0$, and $\varphi_{\tau-t}^{a_{n}}\left(\theta_{-\tau} \omega\right), \widetilde{\varphi}_{\tau-t} \in E$ with $\varphi_{\tau-t}^{a_{n}}\left(\theta_{-\tau} \omega\right) \rightarrow \widetilde{\varphi}_{\tau-t}$, it holds that

$$
\lim _{n \rightarrow \infty} \varphi^{a_{n}}\left(\tau, \tau-t, \theta_{-\tau} \omega, \varphi_{\tau-t}^{a_{n}}\left(\theta_{-\tau} \omega\right)\right)=\widetilde{\varphi}\left(\tau, \tau-t, \widetilde{\varphi}_{\tau-t}\right)
$$

Therefore, $\left\{\mathcal{A}^{a_{n}}(\tau, \omega)\right\}_{a_{n} \in \mathbb{R}}$ and $\{\widetilde{\mathcal{A}}(\tau)\}_{\tau \in \mathbb{R}}$ satisfy the conditions (i)-(iv) of Theorem 2.9 . The proof is completed.

Remark 5.2. If the equation (1.3) satisfies the boundary conditions corresponding to clamped ends

$$
u(x, t)=\nabla u(x, t)=0, \quad x \in \partial U, t \geq \tau,
$$

then we still can prove the existence of a random attractor $\mathcal{A}(\tau, \omega)$ in $H_{0}^{2}(U) \cap L^{2}(U)$ for the RDS defined by (1.3), 5.10 and (1.5).

\section{References}

[1] N. U. Ahmed and H. Harbi, Mathematical analysis of dynamic models of suspension bridges, SIAM J. Appl. Math. 58 (1998), no. 3, 853-874. 
[2] L. Arnold, Random Dynamical Systems, Springer Monographs in Mathematics, Springer-Verlag, Berlin, 1998.

[3] I. Bochicchio, C. Giorgi and E. Vuk, Long-term damped dynamics of the extensible suspension bridge, Int. J. Differ. Equ. 2010 (2010), Art. ID 383420, 19 pp.

[4] I. Chueshov, Monotone Random Systems Theory and Applications, Lecture Notes in Mathematics 1779, Springer-Verlag, Berlin, 2002.

[5] H. Crauel and F. Flandoli, Attractors for random dynamical systems, Probab. Theory Related Fields 100 (1994), no. 3, 365-393.

[6] X. Fan, Random attractor for a damped sine-Gordon equation with white noise, Pacific J. Math. 216 (2004), no. 1, 63-76.

[7] X. Fan and Y. Wang, Fractal dimension of attractors for a stochastic wave equation with nonlinear damping and white noise, Stoch. Anal. Appl. 25 (2007), no. 2, 381-396.

[8] L. D. Humphreys, Numerical mountain pass solutions of a suspension bridge equation, Nonlinear Anal. 28 (1997), no. 11, 1811-1826.

[9] A. C. Lazer and P. J. McKenna, Large-amplitude periodic oscillations in suspension bridges: Some new connections with nonlinear analysis, SIAM Rev. 32 (1990), no. 4, 537-578.

[10] Q. Ma, S. Wang and X. Chen, Uniform compact attractors for the coupled suspension bridge equations, Appl. Math. Comput. 217 (2011), no. 4, 6604-6615.

[11] Q. Ma and C. Zhong, Existence of global attractors for the coupled system of suspension bridge equations, J. Math. Anal. Appl. 308 (2005), no. 1, 365-379.

[12] _ Existence of strong solutions and global attractors for the coupled suspension bridge equations, J. Differential Equations 246 (2009), no. 10, 3755-3775.

[13] W. Ma and Q. Ma, Attractors for stochastic strongly damped plate equations with additive noise, Electron. J. Differential Equations 2013 (2013), no. 111, 12 pp.

[14] P. J. McKenna and W. Walter, Nonlinear oscillations in a suspension bridge, Arch. Rational Mech. Anal. 98 (1987), no. 2, 167-177.

[15] J. Y. Park and J. R. Kang, Pullback $\mathscr{D}$-attractors for non-autonomous suspension bridge equations, Nonlinear Anal. 71 (2009), no. 10, 4618-4623.

[16] - Global attractors for the suspension bridge equations with nonlinear damping, Quart. Appl. Math. 69 (2011), no. 3, 465-475. 
$[17]$ , Uniform attractor for non-autonomous suspension bridge equations with localized damping, Math. Methods Appl. Sci. 34 (2011), no. 4, 487-496.

[18] A. Pazy, Semigroups of Linear Operators and Applications to Partial Differential Equations, Applied Mathematical Sciences 44, Springer-Verlag, New York, 1983.

[19] B. Wang, Upper semicontinuity of random attractors for non-compact random dynamical systems, Electron. J. Differential Equations 2009 (2009), no. 139, 18 pp.

[20] _ Sufficient and necessary criteria for existence of pullback attractors for noncompact random dynamical systems, J. Differential Equations 253 (2012), no. 5, 15441583.

[21] S. Woinowsky-Krieger, The effect of an axial force on the vibration of hinged bars, J. Appl. Mech. 17 (1950), 35-36.

[22] M. Yang, J. Duan and P. Kloeden, Asymptotic behavior of solutions for random wave equations with nonlinear damping and white noise, Nonlinear Anal. Real World Appl. 12 (2011), no. 1, 464-478.

[23] M. Yang and P. E. Kloeden, Random attractors for stochastic semi-linear degenerate parabolic equations, Nonlinear Anal. Real World Appl. 12 (2011), no. 5, 2811-2821.

[24] C. Zhong, Q. Ma and C. Sun, Existence of strong solutions and global attractors for the suspension bridge equations, Nonlinear Anal. 67 (2007), no. 2, 442-454.

\section{Ling Xu}

College of Mathematics and Statistics, Northwest Normal University, Lanzhou, Gansu 730070, China

and

College of Science, National University of Defense Technology, Changsha, Hunan 410073, China

E-mail address: 13893414055@163.com

Qiaozhen Ma

College of Mathematics and Statistics, Northwest Normal University, Lanzhou, Gansu 730070, China

E-mail address: maqzh@nwnu.edu.cn 